\title{
EXISTENCE OF CLASSICAL SOLUTIONS AND FEEDBACK STABILIZATION FOR THE FLOW IN GAS NETWORKS
}

\author{
Martin Gugat $^{1}$ AND Michä̈L Herty ${ }^{2}$
}

\begin{abstract}
We consider the flow of gas through pipelines controlled by a compressor station. Under a subsonic flow assumption we prove the existence of classical solutions for a given finite time interval. The existence result is used to construct Riemannian feedback laws and to prove a stabilization result for a coupled system of gas pipes with a compressor station. We introduce a Lyapunov function and prove exponential decay with respect to the $L^{2}$-norm.
\end{abstract}

Mathematics Subject Classification. 76N25, 35L50, 93C20.

Received March 9, 2009. Revised June 11, 2009.

Published online August 11, 2009.

\section{INTRODUCTION}

The problem of the control of compressor stations in gas networks arises in the daily operation of gas networks and has been studied under a variety of aspects in the recent literature, see for example $[1,2,6,14,27,28,30-34,36]$. The major physical effect in the transportation of gas through a network is the pipe-wall friction which induces a pressure loss along the pipe. The compressors stations are used to increase the pressure. The control objective is twofold. To begin with, the customer demands concerning pressure and flow have to be fulfilled and in addition shock-waves have to be avoided to prevent the material from serious damage. Depending on the model used for predicting the pressure loss, different approaches have been proposed to treat this control objective (see [30] for a comparison). The isothermal Euler equations are considered as the most detailed model, see [31].

In this paper we study the behavior of classical solutions on a pipe network and derive feedback stabilization laws for a network of pipes with a compressor station. The study of classical solutions in this context is motivated by the fact that in a gas pipeline network it is desirable to generate a continuously differentiable state, since non-classical solutions with shocks may damage the pipeline. In this paper we show that for any given finite time interval and sufficiently regular initial data the system state (that is the gas flow) remains continuously differentiable for all $C^{1}$-compatible compressor controls. Moreover, we construct feedback stabilization laws for a network with one compressor station. The construction uses a strict Lyapunov function for the network and is stated in terms of Riemann invariants. Our construction is based upon the ideas stated in $[7,11,12]$ for the case of a system without source terms. However, for our network, the source terms are essential. On account of the source terms, the stationary states of our system are not constant. In order to take this fact into account, we have generalized the construction given in [7] to the case of non-constant equilibria.

\footnotetext{
Keywords and phrases. Classical solution, networked hyperbolic systems, gas networks, feedback law, Lyapunov function.

1 Lehrstuhl 2 für Angewandte Mathematik, Martensstr. 3, 91058 Erlangen, Germany. gugat@am.uni-erlangen.de

2 RWTH Aachen, Lehrstuhl C für Mathematik, Templergraben 55, 52065 Aachen, Germany. herty@mathc.rwth-aachen.de
} 


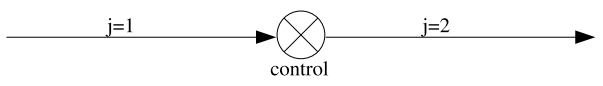

FiguRE 1. Illustration of the control of a compressor station with two pipes. The first pipe corresponds to the supplier and the second to the customer.

The problem of stabilization and control of gas networks is similar to the problem of flow control in open channels. Networks of open channels modeled by the Saint-Venant equations have been studied for example in $[4,7-11,13,15]$. The corresponding results of exact boundary controllability are related with [3]. However, there are some important differences concerning the equation itself, the type of control which is applied, the source terms and the control objective. In fact, for the Saint-Venant equations the source term has a more complex structure than for the system studied in this paper which leads to different stationary states, see [16]. For the water flow in channel networks, also supercritical flow is of interest, see [17].

This paper is organized as follows. We review the model for gas flow and compressor control in pipe networks in Section 1 and present our main results. In Section 3 we rewrite the system and the compressor condition in terms of Riemann invariants and consider the linearization around stationary states (stationary states are analyzed in Sect. 2). Let us emphasize again that due to the source term the stationary states are not constant and exist as continuously differentiable functions only on a finite space interval. The semi-global existence result for classical solutions is given in Sections 4.1 and 4.2. Finally, the stabilization result with the corresponding feedback law is given in Section 5 .

\section{MAin RESUlts FOR A MODEL OF GAS FLOW IN PIPE NETWORKS}

A common model for gas flow in pipe networks are the isothermal Euler equations $[1,2,5,6,14,18,28,29]$. In a single pipe the gas flow is described by the equations

$$
\begin{aligned}
\rho_{t}+q_{x} & =0 \\
q_{t}+\left(\frac{q^{2}}{\rho}+a^{2} \rho\right)_{x} & =-f_{g} \frac{q|q|}{2 D \rho}
\end{aligned}
$$

where $\rho(t, x)$ is the density of the gas, $q(t, x)$ is the mass flux in the pipe, $f_{g}$ is the friction factor and $D$ is the diameter of the pipe. The first equation states the conservation of mass and the second equation is the momentum equation. The pressure law is

$$
p=a^{2} \rho \text { where } a^{2}=\frac{Z \mathcal{R} \mathcal{T}}{M_{g}},
$$

and $Z$ is the natural gas compressibility factor, $\mathcal{R}$ the universal gas constant, $\mathcal{T}$ the absolute gas temperature and $M_{g}$ is the gas molecular weight, see [29]. Equations (1.1a), (1.1b) form the $p$-system which is a hyperbolic balance law.

In order to treat networks of pipes controlled by compressors we consider a simple network consisting of two pipes parametrized by the intervals $\left[0, L_{1}\right]$ and $\left[0, L_{2}\right]$ and connected at $x=L_{1}$ and $x=0 \in\left[0, L_{2}\right]$ respectively by a compressor station as depicted in Figure 1. Let a time horizon $T>0$ be given.

If we consider $C^{1}$-solutions, using the notation

$$
\begin{aligned}
& y=\left(\begin{array}{c}
\rho \\
q
\end{array}\right), \hat{G}(y)=\left(\begin{array}{c}
0 \\
-f_{g} \frac{q|q|}{2 D \rho}
\end{array}\right), \\
& F(y)=\left(\begin{array}{c}
q \\
\frac{q^{2}}{\rho}+a^{2} \rho
\end{array}\right), \hat{A}=D_{y} F(y)=\left(\begin{array}{cc}
0 & 1 \\
a^{2}-\frac{q^{2}}{\rho^{2}} & 2 \frac{q}{\rho}
\end{array}\right)
\end{aligned}
$$


the dynamics (1.1) for the state $y^{(1)}$ on the first pipe and the state $y^{(2)}$ on the second pipe can be rewritten as

$$
\begin{aligned}
& \partial_{t} y^{(1)}+\hat{A}\left(y^{(1)}\right) \partial_{x} y^{(1)}=\hat{G}\left(y^{(1)}\right) \text { on } \mathcal{D}^{(1)} \\
& \partial_{t} y^{(2)}+\hat{A}\left(y^{(2)}\right) \partial_{x} y^{(2)}=\hat{G}\left(y^{(2)}\right) \text { on } \mathcal{D}^{(2)}
\end{aligned}
$$

where the space-time rectangles $\mathcal{D}^{(1)}, \mathcal{D}^{(1)}$ for the pipes are defined as

$$
\mathcal{D}^{(1)}=\left\{(t, x): t \in[0, T], 0 \leq x \leq L_{1}\right\}, \mathcal{D}^{(2)}=\left\{(t, x): t \in[0, T], 0 \leq x \leq L_{2}\right\} .
$$

The two pipes are coupled at $x=L_{1}$ and $x=0$, respectively, by a compressor: By applying a certain compressor power $u \geq 0$ the compressors increases the outlet pressure according to the following nonlinear conditions (see $[14,19])$

$$
\begin{aligned}
q^{(1)}\left(t, L_{1}\right) & =q^{(2)}(t, 0) \\
u(t) & =q^{(2)}(t, 0)\left(\left(\frac{\rho^{(2)}(t, 0)}{\rho^{(1)}\left(t, L_{1}\right)}\right)^{\kappa}-1\right) .
\end{aligned}
$$

This model is only valid if the flow in the pipe satisfies the inequality $q^{(2)}(t, 0) \geq 0$ that is if the gas flows from the first pipe into the second pipe. The power $\kappa$ depends on the gas under consideration and we have $\kappa \in\left[\frac{1}{3}, \frac{3}{5}\right]$.

In Section 3 the system $(1.4 \mathrm{a}, 1.4 \mathrm{~b}, 1.5 \mathrm{a}, 1.5 \mathrm{~b})$ is rewritten in terms of its Riemann invariants $R_{ \pm}^{(1)}, R_{ \pm}^{(2)}$. It is equivalent to a diagonal system of the form $(i \in\{1,2\}$, see (3.8), (3.12))

$$
\begin{aligned}
\partial_{t}\left(\begin{array}{c}
R_{+}^{(i)} \\
R_{-}^{(i)}
\end{array}\right)+\left(\begin{array}{cc}
-\frac{R_{+}^{(i)}+R_{-}^{(i)}}{2}+a & 0 \\
0 & -\frac{R_{+}^{(i)}+R_{-}^{(i)}}{2}-a
\end{array}\right) \partial_{x}\left(\begin{array}{c}
R_{+}^{(i)} \\
R_{-}^{(i)}
\end{array}\right) & =-\frac{f_{g}}{2 D} \frac{\left(R_{+}^{(i)}+R_{-}^{(i)}\right)}{2} \frac{\left|R_{+}^{(i)}+R_{-}^{(i)}\right|}{2}\left(\begin{array}{c}
1 \\
1
\end{array}\right), \\
R_{+}^{(2)}(t, 0) & =\Psi_{2}\left(R_{+}^{(1)}\left(t, L_{1}\right), R_{-}^{(2)}(t, 0), u(t)\right) \\
R_{-}^{(1)}\left(t, L_{1}\right) & =\Psi_{1}\left(R_{+}^{(1)}\left(t, L_{1}\right), R_{-}^{(2)}(t, 0), u(t)\right)
\end{aligned}
$$

and initial conditions $R_{ \pm}^{(i)}(0, x)=R_{0, \pm}^{(i)}(x)(i \in\{1,2\})$ and suitable boundary conditions (see Sect. 3.3). Here for $i \in\{1,2\}$ in the diagonal system matrix the eigenvalues

$$
\lambda_{+}^{(i)}=-\frac{R_{+}^{(i)}+R_{-}^{(i)}}{2}+a, \lambda_{-}^{(i)}=-\frac{R_{+}^{(i)}+R_{-}^{(i)}}{2}-a
$$

appear. Let $D\left(R^{(i)}\right)$ denote the corresponding $2 \times 2$ diagonal matrix. Theorem 4.1 states that for a given stationary $x$-dependent solution $\bar{R}_{ \pm}^{(i)}$ with the constant control $\bar{u}$ (see Sect. 2) there exists a solution $r_{ \pm}^{(i)}(x, t)$ $(i \in\{1,2\})$ of the perturbed system

$$
\begin{aligned}
\partial_{t} r^{(i)}+D\left(r^{(i)}+\bar{R}^{(i)}\right) \partial_{x} r^{(i)}=\tilde{G}^{(i)}\left(\bar{R}^{(i)}, r^{(i)}\right), & \\
\Psi_{1}\left(r_{+}^{(1)}\left(t, L_{1}\right)+\bar{R}_{+}^{(1)}\left(L_{1}\right), r_{-}^{(2)}(t, 0)+\bar{R}_{-}^{(2)}(0), \bar{u}+v(t)\right)-\bar{R}_{-}^{(1)}\left(L_{1}\right) & =r_{-}^{(1)}\left(t, L_{1}\right) \\
\Psi_{2}\left(r_{+}^{(1)}\left(t, L_{1}\right)+\bar{R}_{+}^{(1)}\left(L_{1}\right), r_{-}^{(2)}(t, 0)+\bar{R}_{-}^{(2)}(0), \bar{u}+v(t)\right)-\bar{R}_{+}^{(2)}(0) & =r_{+}^{(2)}(t, 0)
\end{aligned}
$$

that satisfies the initial conditions $r^{(i)}(0, x)=b^{(i)}(x)$ if the $C^{1}$-norms of $b^{(i)}$ and $v$ are sufficiently small. Clearly, $R_{ \pm}^{(i)}=\bar{R}_{ \pm}^{(i)}+r_{ \pm}^{(i)}(t, x)$ or equivalently $y^{(i)}=\left(\rho^{(i)}, q^{(i)}\right)$ is then a solution to the original problem.

In Section 4.2 we establish a global existence result: let $T>0$ and $\gamma>0$ be given. There exists a real number $\varepsilon>0$ such that for all subsonic stationary initial $C^{1}$-states with constant flow rate $\bar{q}>0$ and boundary 
density $\rho^{(1)}(t, 0)=\gamma \bar{q}$ at $x=0$ in the first pipe and all control functions $u(t) \geq 0$ satisfying $u(0)=0$ and the $C^{1}$-compatibility conditions at the compressor node and the inequality

$$
\frac{\|u\|_{C^{1}([0, T])}}{\bar{q}} \leq \varepsilon
$$

there exist $C^{1}$-solutions $\left(\rho^{(i)}, q^{(i)}\right)(t, x)$ of the pipeline-compressor system on the sets $\mathcal{D}^{(i)}, i \in\{1,2\}$ respectively with compatible constant boundary densities. Note that in the above inequality, the values of $u(t)$ and $\bar{q}$ are allowed to be large as long as their quotient is sufficiently small.

Concerning the stabilization around the stationary state $\bar{R}_{ \pm}^{(i)}, i \in\{1,2\}$ we establish the following result with precise assumptions given in Theorem 5.3.

Theorem 1.1. Consider the closed loop system, where at the compressor node the control function $u(t)$ is chosen in such a way that $q(t)=\bar{q}$, that is the compressor power maintains a steady mass flux. Hence, the control law is given by

$$
u(t)=\bar{q}\left(\left(\frac{\rho^{(2)}(t, 0)}{\rho^{(1)}\left(t, L_{1}\right)}\right)^{\kappa}-1\right) .
$$

We assume that at the boundary node $x=0$ of the first pipe we have a linear feedback law

$$
r_{+}^{(1)}(t, 0)=k^{(1)} r_{-}^{(1)}(t, 0)
$$

with a real constant $k^{(1)}$. Moreover we assume that at the boundary node of the second pipe we have a linear feedback law of the same form, $r_{-}^{(2)}\left(t, L_{2}\right)=k^{(2)} r_{+}^{(2)}\left(t, L_{2}\right)$.

Assume that the constants $k^{(1)}, k^{(2)}$ in the linear boundary feedback laws satisfy the assumptions of Theorem 5.3 and that a $C^{1}$-solution $\left(r^{(1)}, r^{(2)}\right)$ exists on the time interval $[0, T]$ in the pipeline network for the closed loop system and has sufficiently small $C^{1}$-norm. For $i \in\{1,2\}$, define the functions

$$
\bar{\lambda}_{+}^{(i)}=-\frac{\bar{R}_{+}^{(i)}+\bar{R}_{-}^{(i)}}{2}+a, \bar{\lambda}_{-}^{(i)}=-\frac{\bar{R}_{+}^{(i)}+\bar{R}_{-}^{(i)}}{2}-a
$$

and for $\mu^{(i)}>0$, let

$$
h_{+}^{(i)}(x)=\exp \left(-\mu^{(i)} \int_{0}^{x} \frac{1}{\bar{\lambda}_{+}^{(i)}(s)} \mathrm{d} s\right), \quad h_{-}^{(i)}(x)=\exp \left(\mu^{(i)} \int_{0}^{x} \frac{1}{-\bar{\lambda}_{-}^{(i)}(s)} \mathrm{d} s\right) .
$$

We define the network Lyapunov function

$$
E_{N}(t)=\sum_{i=1}^{2} \int_{0}^{L_{i}}\left[\frac{A^{(i)}}{\bar{\lambda}_{+}^{(i)}(x)}\left[r_{+}^{(i)}(t, x)\right]^{2} h_{+}^{(i)}(x)+\frac{B^{(i)}}{-\bar{\lambda}_{-}^{(i)}(x)}\left[r_{-}^{(i)}(t, x)\right]^{2} h_{-}^{(i)}(x)\right] \mathrm{d} x .
$$

If the constants $A^{(i)}>0, B^{(i)}>0$ and $\mu^{(i)}$ are chosen in such a way that the assumptions of Theorem 5.3 hold and the pipes are sufficiently short, the function $E_{N}(t)$ decays exponentially on the time-interval $[0, T]$.

\section{Stationary states For a Network of isothermal Euler EQUATions}

In this section we consider stationary subsonic continuously differentiable states. Due to the source-term, for our system the stationary states are not constant. In general, they exist as classical solutions only on a finite (space-) interval until a singularity of the derivative occurs. For the representation of the stationary states, we need the following lemma. 
Lemma 2.1. Let the real number $\bar{q} \geq 0$ be given. Define the interval $I=[|\bar{q}| / a, \infty)$. For $z \in I$, define the function $F(z)=a^{2} z^{2}-2 \bar{q}^{2} \log (z)$. Then for all $z \in(|\bar{q}| / a, \infty), F^{\prime}(z)>0$, hence $F$ is strictly increasing on $I$. Moreover, $F^{\prime \prime}(z)>0$, thus $F$ is strictly convex on $I$. We have $F^{\prime}(|\bar{q}| / a)=0$ and $\lim _{z \rightarrow \infty} F(z)=\infty$. Define

$$
a_{0}=F(|\bar{q}| / a)=\bar{q}^{2}\left(1-\log \left((\bar{q} / a)^{2}\right)\right) .
$$

Then the inverse function $H(t):=F^{-1}(t)$ is well-defined and strictly increasing and strictly concave on $\left[a_{0}, \infty\right)$. Moreover, we have $H\left(a_{0}\right)=|\bar{q}| / a$,

$$
\lim _{t \rightarrow a_{0}+} H^{\prime}(t)=\lim _{z \rightarrow|\bar{q}| / a} \frac{1}{F^{\prime}(z)}=\infty
$$

and $\lim _{t \rightarrow \infty} H(t)=\infty, \lim _{t \rightarrow \infty} H^{\prime}(t)=0$

Now we construct stationary solutions $\bar{y}(x)=(\bar{\rho}(x), \bar{q})^{T}$ to the isothermal Euler equations. Clearly, $\bar{q}$ is just a constant and $\bar{\rho}$ is a solution of the ordinary differential equation

$$
\frac{\mathrm{d}}{\mathrm{d} x}\left(a^{2} \bar{\rho}+\frac{\bar{q}^{2}}{\bar{\rho}}\right)=-f_{g} \frac{\bar{q}|\bar{q}|}{2 D \bar{\rho}} .
$$

Since $\bar{\rho}>0$, we can write this equation as

$$
\frac{\mathrm{d}}{\mathrm{d} x}\left(a^{2} \bar{\rho}^{2}-2 \bar{q}^{2} \log (\bar{\rho})\right)=-f_{g} \frac{\bar{q}|\bar{q}|}{D}
$$

so that $\bar{\rho}(x)$ is implicitly defined by

$$
a^{2} \bar{\rho}^{2}(x)-2 \bar{q}^{2} \log (\bar{\rho}(x))=a^{2} \bar{\rho}_{0}^{2}-2 \bar{q}^{2} \log \left(\bar{\rho}_{0}\right)-f_{g} \frac{\bar{q}|\bar{q}|}{D} \cdot x,
$$

where $\bar{\rho}_{0}=\bar{\rho}(0)>0$. We assume that we have a subsonic state that is

$$
\bar{q} / \bar{\rho}_{0}<a,
$$

hence $\bar{\rho}_{0}>\bar{q} / a$, thus $F\left(\bar{\rho}_{0}\right)$ is well-defined and $\bar{\rho}_{0}$ is in the interior of the interval $I$ as defined in Lemma 2.1 . If $\bar{\rho}(x) \in I$, we have $F(\bar{\rho}(x))=a^{2} \bar{\rho}_{0}^{2}-2 \bar{q}^{2} \log \left(\bar{\rho}_{0}\right)-f_{g} \frac{\bar{q}|\bar{q}|}{D} \cdot x$ which implies the equation

$$
\bar{\rho}(x)=H\left(a^{2} \bar{\rho}_{0}^{2}-2 \bar{q}^{2} \log \left(\bar{\rho}_{0}\right)-f_{g} \frac{\bar{q}|\bar{q}|}{D} \cdot x\right),
$$

with $H$ as defined in Lemma 2.1. Since the function $H$ is strictly increasing, this implies that the density $\bar{\rho}$ is strictly decreasing along the pipe provided that $\bar{q}>0$.

Assume that $\bar{q}>0$. Define the critical length

$$
x_{0}=D \frac{a^{2} \bar{\rho}_{0}^{2}-2 \bar{q}^{2} \log \left(\bar{\rho}_{0}\right)-a_{0}}{f_{g} \bar{q}|\bar{q}|}=D \frac{F\left(\bar{\rho}_{0}\right)-a_{0}}{f_{g} \bar{q}|\bar{q}|}>0 .
$$

Then we have

$$
\lim _{x \rightarrow x_{0}} \bar{\rho}(x)=H\left(a_{0}\right)=|\bar{q}| / a,
$$

hence for $x \rightarrow x_{0}$ the state becomes critical. This implies in particular that the state remains subsonic along the pipe on the interval $\left[0, x_{0}\right)$. Equation (2.2) implies that for $x \rightarrow x_{0}$ - we have $\bar{\rho}(x)^{\prime} \rightarrow-\infty$, hence for the stationary solutions after the critical length blow up in the derivative occurs and the solution cannot be extended as a $C^{1}$-solution beyond this length. This implies that stationary $C^{1}$-solutions exist on the whole pipe if and only if its length $L$ is less than the critical length, that is if $L<x_{0}$. 
Next, we consider the case as in Section 1 where our system consists of two pipes coupled through a compressor. On each pipe, we have a constant flux $\bar{q}$ and due to the conservation of mass coupling conditions (1.5a) we have the same constant flux $\bar{q}$ on both pipes. Assume that $\bar{q}>0$. This is no restriction to real world applications since compressor stations work only in one direction and changes of the flux directions do not occur. On each pipe (that is for $i \in\{1,2\}$ ) the density $\bar{\rho}_{i}(x)$ is given by (2.6).

Let $\bar{\rho}_{0}=\bar{\rho}_{1}(0)$ be the density at the inflow point of the first pipe, that corresponds to the interval $\left[0, L_{1}\right]$. Let the number $a_{0}$ be as in (2.1).

To make sure that on the first pipe a $C^{1}$-stationary state exists, the condition

$$
L_{1}<x_{0}
$$

with $x_{0}$ as in (2.7) must hold. This means that the density $\bar{\rho}_{0}$ at the inflow point must be sufficiently large. Then $\bar{\rho}_{1}(x)$ is well-defined as a $C^{1}$-function on the interval $\left[0, L_{1}\right]$ that corresponds to the first pipe. The second coupling condition $(1.5 \mathrm{~b})$ implies that $\bar{\rho}_{2}(0)=\bar{\rho}_{1}\left(L_{1}\right)[u / \bar{q}+1]^{1 / \kappa}$, where $u \geq 0$ is the constant compressor control. Define the real number $\tilde{\rho}_{0}=\bar{\rho}_{2}(0)$. A $C^{1}$-stationary state exists on the second pipe if and only if the condition

$$
L_{2}<\tilde{x}_{0}=D \frac{a^{2} \tilde{\rho}_{0}^{2}-2 \bar{q}^{2} \log \left(\tilde{\rho}_{0}\right)-a_{0}}{f_{g} \bar{q}|\bar{q}|}=D \frac{F\left(\tilde{\rho}_{0}\right)-a_{0}}{f_{g} \bar{q}|\bar{q}|}
$$

is valid. If it holds, $\bar{\rho}_{2}(x)$ is a well-defined $C^{1}$-function on $\left[0, L_{2}\right]$.

If (2.8) and (2.9) hold, we have a $C^{1}$-stationary state in our system. Note that through the definition of $\tilde{\rho}_{0}$ inequality (2.9) is a condition on the compressor pressure $u$. Condition (2.9) is valid if $u$ is sufficiently large. If (2.9) holds for $u=0$, it is also valid for all $u>0$. This is a consequence of the fact that the function $F$ defined in Lemma 2.1 appears in the numerator, and in Lemma 2.1 we have seen that $F$ is strictly increasing, and $\tilde{\rho}_{0}$ is also strictly increasing as a function of $u$.

We have seen that the $C^{1}$-stationary states are uniquely determined by the values $\bar{q}>0, \bar{\rho}_{0}>0$ and $u>0$, provided that (2.8) and (2.9) hold. While (2.8) can always be checked explicitly, the verification of (2.9) is more involved since it requires the computation of $\bar{\rho}_{1}\left(L_{1}\right)$. A simple sufficient condition for the existence of $C^{1}$-stationary states for all $u \geq 0$ is the inequality $L_{1}+L_{2}<x_{0}$, that implies that (2.8) is valid and (2.9) holds for $u=0$ and thus also for all $u \geq 0$.

As a result of our discussion we give a simple sufficient condition for the existence of a stationary state on our network of two pipes from Section 1.

Lemma 2.2. Let $\bar{q}>0$ and $\bar{\rho}_{0}>0$ be given such that $\bar{q}<a \bar{\rho}_{0}$. Define the number $a_{0}$ as in (2.1) and the critical length $x_{0}$ as in (2.7). Assume that the inequality

$$
L_{1}+L_{2}<x_{0}
$$

holds. Then for all constant controls $u \geq 0$ a subsonic $C^{1}$-stationary solution exists in our network with the constant flow rate $\bar{q}$ and the boundary value $\rho^{(1)}(t, 0)=\bar{\rho}_{0}$. It satisfies the isothermal Euler equations along the pipes and the coupling conditions (1.5a), (1.5b) in the compressor.

\section{TRANSFormation to RiEMAnN InVARiants}

In order to prove the semi-global existence of classical solutions we reformulate the dynamics on each pipe $(1.4 \mathrm{a}, 1.4 \mathrm{~b})$ in terms of Riemann invariants. Since the equations are the same for each pipe and in order to improve the readability we skip the upper index in the following derivation and study the equation

$$
\partial_{t} y+\hat{A}(y) \partial_{x} y=\hat{G}(y)
$$


We will frequently switch between the variable $y$ and the pair of $\rho$ and $q$. A state is called subsonic, if

$$
\left|\frac{q}{\rho}\right|<a
$$

with the number $a$ defined in (1.2).

\subsection{The Riemann invariants of the system}

For subsonic states $\hat{A}(y)$ has two eigenvalues $\lambda_{ \pm}(y)=\frac{q}{\rho} \pm a$ with the property $\lambda_{-}<0<\lambda_{+}$and we obtain

$$
\hat{A}(y)=\left(\begin{array}{cc}
0 & 1 \\
-\lambda_{-} \lambda_{+} & \lambda_{+}+\lambda_{-}
\end{array}\right) .
$$

The corresponding left and right eigenvectors are given by

$$
\begin{aligned}
& l_{ \pm}(y)=\left(\frac{q}{\rho^{2}} \mp \frac{a}{\rho},-\frac{1}{\rho}\right)=\frac{1}{\rho}\left(\lambda_{\mp},-1\right), \\
& r_{ \pm}(y)=\left(\begin{array}{c}
\rho \\
q \pm a \rho
\end{array}\right)=\rho\left(\begin{array}{c}
1 \\
\lambda_{ \pm}
\end{array}\right) .
\end{aligned}
$$

Denote by $L(y)$ the matrix of left eigenvectors, then $\operatorname{det}(L(y))=\frac{1}{\rho^{2}}\left(\lambda_{+}-\lambda_{-}\right)=2 \frac{a}{\rho^{2}} \neq 0$. We obtain the Riemann invariants as

$$
R_{ \pm}(y)=-\frac{q}{\rho} \mp a \ln (\rho)
$$

and restate the eigenvalues $\lambda_{ \pm}, \rho$ and $q$ in terms of $R_{ \pm}$as

$$
\lambda_{ \pm}=-\frac{R_{+}+R_{-}}{2} \pm a, \rho=\exp \left(\frac{R_{-}-R_{+}}{2 a}\right), q=-\frac{R_{+}+R_{-}}{2} \exp \left(\frac{R_{-}-R_{+}}{2 a}\right) .
$$

For subsonic states we have $\left|R_{+}+R_{-}\right|<2 a$ and

$$
\frac{\partial q}{\partial R_{+}}<0, \frac{\partial q}{\partial R_{-}}<0
$$

and for $\rho>\exp (0)=1$ we have the inequality $R_{+}<R_{-}$. Define the function

$$
Q\left(R_{+}, R_{-}\right)=-\frac{R_{+}+R_{-}}{2} \exp \left(\frac{R_{-}-R_{+}}{2 a}\right) .
$$

Due to the monotonicity properties there exist differentiable functions $F_{+}, F_{-}$such that for all fixed real values of $R_{+}, q^{*}$ with $q^{*} \in\left(-a \exp \left(1-R_{+} / a\right), a \exp \left(-1-R_{+} / a\right)\right)=\left(Q\left(R_{+}, 2 a-R_{+}\right), Q\left(R_{+},-2 a-R_{+}\right)\right)$we have the equation

$$
Q\left(R_{+}, F_{-}\left(q^{*}, R_{+}\right)\right)=q^{*},
$$

and for all fixed $R_{-}, q^{*}$ with $q^{*} \in\left(-a \exp \left(-1+R_{-} / a\right), a \exp \left(1+R_{-} / a\right)\right)=\left(Q\left(2 a-R_{-}, R_{-}\right), Q\left(-2 a-R_{-}\right)\right)$ we have the equation

$$
Q\left(F_{+}\left(q^{*}, R_{-}\right), R_{-}\right)=q^{*}
$$

and the states $\left(R_{+}, F_{-}\left(q^{*}, R_{+}\right)\right),\left(F_{+}\left(q^{*}, R_{-}\right), R_{-}\right)$are subsonic. 
We have $\partial_{R_{+}} F_{-}=\frac{\frac{q}{\rho}+a}{\frac{q}{\rho}-a}<0$ and $\partial_{R_{-}} F_{+}=\frac{\frac{q}{\rho}-a}{\frac{q}{\rho}+a}<0$ hence the functions $F_{-}, F_{+}$are strictly decreasing with respect to $R_{+}, R_{-}$respectively. Moreover,

$$
\partial_{q} F_{+}=\frac{2}{-\rho-\frac{q}{a}}<0, \quad \partial_{q} F_{-}=\frac{2}{-\rho+\frac{q}{a}}<0
$$

and

$$
F_{+}\left(0, R_{-}\right)=-R_{-}, \quad F_{-}\left(0, R_{+}\right)=-R_{+}
$$

\subsection{The compressor condition in terms of Riemann invariants}

In this section we show that for given values $u(t), R_{+}^{(1)}$ and $R_{-}^{(2)}$ satisfying (3.6) we can express the compressor conditions $(1.5 \mathrm{a}, 1.5 \mathrm{~b})$ in the form

$$
\left(\begin{array}{l}
R_{-}^{(1)}\left(t, L_{1}\right) \\
R_{+}^{(2)}(t, 0)
\end{array}\right)=\left(\begin{array}{c}
\Psi_{1}\left(R_{+}^{(1)}\left(t, L_{1}\right), R_{-}^{(2)}(t, 0), u(t)\right) \\
\Psi_{2}\left(R_{+}^{(1)}\left(t, L_{1}\right), R_{-}^{(2)}(t, 0), u(t)\right)
\end{array}\right) .
$$

Due to the previous calculations for fixed $q^{*} \geq 0, R_{+}^{(1)}, R_{-}^{(2)}$ we have the equations

$$
R_{-}^{(1)}=F_{-}\left(q^{*}, R_{+}^{(1)}\right), R_{+}^{(2)}=F_{+}\left(q^{*}, R_{-}^{(2)}\right)
$$

and obtain a subsonic state that satisfies the condition (1.5a) and

$$
R_{-}^{(1)} \leq F_{-}\left(0, R_{+}^{(1)}\right)=-R_{+}^{(1)}, R_{+}^{(2)} \leq F_{+}\left(0, R_{-}^{(2)}\right)=-R_{-}^{(2)}
$$

We have

$$
\begin{aligned}
\frac{\rho^{(2)}}{\rho^{(1)}} & =\frac{\exp \left(\frac{R_{-}^{(2)}-F_{+}\left(q^{*}, R_{-}^{(2)}\right)}{2 a}\right)}{\exp \left(\frac{F_{-}\left(q^{*}, R_{+}^{(1)}\right)-R_{+}^{(1)}}{2 a}\right)} \\
& =\exp \left(\frac{R_{+}^{(1)}+R_{-}^{(2)}-F_{+}\left(q^{*}, R_{-}^{(2)}\right)-F_{-}\left(q^{*}, R_{+}^{(1)}\right)}{2 a}\right)=A_{0}\left(q^{*}\right)
\end{aligned}
$$

Then for any subsonic state we have $A_{0}^{\prime}\left(q^{*}\right)>0$. Moreover,

$$
A_{0}(0)=\exp \left(\frac{R_{-}^{(2)}+R_{+}^{(1)}}{a}\right) \text {. }
$$

Assume that $A_{0}(0)<1$, that is

$$
R_{+}^{(1)}+R_{-}^{(2)}<0
$$

Lemma 3.1 gives a sufficient condition for the inequality (3.6). Let $q_{1}=\sup \left\{q \geq 0: A_{0}(q)<1\right\}$. Then $q_{1}>0$. Note that with the flow rate $q_{1}$ at the compressor we have $\rho^{(1)}=\rho^{(2)}$.

We can write the second compressor condition $(1.5 \mathrm{~b})$ in the form

$$
A_{1}\left(q^{*}\right):=\left(A_{0}\left(q^{*}\right)^{\kappa}-1\right) q^{*}=u(t)
$$


We have $A_{1}(0)=0, A_{1}\left(q_{1}\right)=0$ and

$$
A_{1}^{\prime}\left(q^{*}\right)=\left(A_{0}\left(q^{*}\right)^{\kappa}-1\right)+q^{*} \kappa A_{0}\left(q^{*}\right)^{\kappa-1} A_{0}^{\prime}\left(q^{*}\right),
$$

hence $A_{1}^{\prime}(0)<0, A_{1}^{\prime}\left(q_{1}\right)=q_{1} \kappa A_{0}^{\prime}\left(q_{1}\right)>0$ and for all values $q^{*} \geq q_{1}$ close to $q_{1}$ we have $A_{1}^{\prime}\left(q^{*}\right)>0$.

Therefore for all sufficiently small values $u(t) \geq 0$ we can find a unique value $q^{*} \geq q_{1}$ such that (3.7) holds.

This value $q^{*}$ depends continuously differentiable on $u(t), R_{+}^{(1)}$ and $R_{-}^{(2)}$.

If $u(t)=0$ we have in general two solutions that satisfy the compressor equations, namely the solution $q=q_{1}$, $A_{0}\left(q_{1}\right)=1$ and $q=0$ as a second solution. In this case we set $q=q_{1}$.

Then, for all $u(t) \geq 0$, the previous construction yields a value for $q^{*} \geq q_{1}$ and we can define $R_{-}^{(1)}, R_{+}^{(2)}$ by (3.5) for this particular $q^{*}$. Then, we have a solution of the compressor equations (1.5a), (1.5b).

Hence, for data satisfying (3.6), that is with $R_{+}^{(1)}+R_{-}^{(2)} \leq 0$ the compressor equations (1.5a), (1.5b) can be reformulated as

$$
\left(\begin{array}{l}
R_{-}^{(1)}\left(t, L_{1}\right) \\
R_{+}^{(2)}(t, 0)
\end{array}\right)=\left(\begin{array}{c}
\Psi_{1}\left(R_{+}^{(1)}\left(t, L_{1}\right), R_{-}^{(2)}(t, 0), u(t)\right) \\
\Psi_{2}\left(R_{+}^{(1)}\left(t, L_{1}\right), R_{-}^{(2)}(t, 0), u(t)\right)
\end{array}\right)
$$

with $C^{1}$-functions $\Psi_{1}, \Psi_{2}$.

In the next lemma we give a sufficient condition for (3.6) in terms of the physical variables, which states that (3.6) holds if the control values are sufficiently small.

Lemma 3.1. Assume that $q>0$ and

$$
u(t) \in\left[0, q\left\{\left[\exp \left(\frac{q}{a \rho^{(1)}}\right)\right]^{\kappa}-1\right\}\right] .
$$

Then (3.6) holds.

Proof. We have $R_{-}^{(2)}=-q / \rho^{(2)}+a \ln \left(\rho^{(2)}\right), R_{+}^{(1)}=-q / \rho^{(1)}-a \ln \left(\rho^{(1)}\right)$. Hence our desired inequality is equivalent to

$$
\ln \left(\frac{\rho^{(2)}}{\rho^{(1)}}\right)<\frac{q}{a}\left[\frac{1}{\rho^{(1)}}+\frac{1}{\rho^{(2)}}\right]=\frac{q}{a \rho^{(1)}}\left(1+\frac{1}{\rho^{(2)} / \rho^{(1)}}\right) .
$$

Due to the compressor equation (1.5b), our assumption on $u$ implies that

$$
\frac{\rho^{(2)}}{\rho^{(1)}}=\left(1+\frac{u}{q}\right)^{1 / \kappa} \leq \exp \left(\frac{q}{a \rho^{(1)}}\right)
$$

Define the function $H(z)=\frac{\ln (z)}{1+1 / z}=\frac{z \ln (z)}{1+z}$. Then $H$ is strictly increasing on the interval $[1, \infty)$. Hence we have the inequality

$$
H\left(\frac{\rho^{(2)}}{\rho^{(1)}}\right) \leq H\left(\exp \left(\frac{q}{a \rho^{(1)}}\right)\right)<\frac{q}{a \rho^{(1)}}
$$

and inequality (3.10) and thus the assertion follows.

\subsection{Boundary conditions in terms of Riemann invariants}

The boundary conditions at the end of the first pipe at $x=0$ and at $x=L_{2}$ for the second pipe respectively can be rewritten in terms of Riemann invariants. Typical boundary conditions for gas flow are either flow or density conditions. A boundary condition of the form $Q(t, 0)=q_{1}(t)$ where the flow rate is prescribed can be written as $R_{+}(t, 0)=F_{+}\left(q_{1}(t), R_{-}(t, 0)\right)$. Analogously, a boundary condition of the form $Q\left(t, L_{2}\right)=q_{2}(t)$ can be written as $R_{-}\left(t, L_{2}\right)=F_{-}\left(q_{2}(t), R_{+}\left(t, L_{2}\right)\right)$. 
A boundary condition of the form $\rho(t, 0)=\rho_{1}(t)$ where the density is prescribed can be written as

$$
R_{+}(t, 0)=R_{-}(t, 0)-2 a \ln \left(\rho_{1}(t)\right)
$$

or analogously $\rho\left(t, L_{2}\right)=\rho_{2}(t)$ can be transformed to $R_{-}\left(t, L_{2}\right)=R_{+}\left(t, L_{2}\right)+2 a \ln \left(\rho_{2}(t)\right)$.

\subsection{The system in Riemann invariants and its representation around a stationary state}

Now we write our system in terms of Riemann invariants $\left(R_{+}, R_{-}\right)$as unknown functions and obtain the corresponding diagonal system matrix $D\left(R_{+}, R_{-}\right)$as

$$
D\left(R_{+}, R_{-}\right)=\left(\begin{array}{cc}
\lambda_{+} & 0 \\
0 & \lambda_{-}
\end{array}\right)=\left(\begin{array}{cc}
-\frac{R_{+}+R_{-}}{2}+a & 0 \\
0 & -\frac{R_{+}+R_{-}}{2}-a
\end{array}\right) .
$$

The source term $\hat{G}$ is also rewritten in Riemann invariants and given by

$$
G\left(R_{+}, R_{-}\right)=\left(\begin{array}{c}
l_{+} \hat{G} \\
l_{-} \hat{G}
\end{array}\right)=\left(\begin{array}{c}
\frac{f_{g}}{2 D} \frac{q|q|}{\rho^{2}} \\
\frac{f_{g}}{2 D} \frac{q|q|}{\rho^{2}}
\end{array}\right)=-\frac{f_{g}}{2 D} \frac{\left(R_{+}+R_{-}\right)}{2} \frac{\left|R_{+}+R_{-}\right|}{2}\left(\begin{array}{c}
1 \\
1
\end{array}\right) .
$$

Finally, for a single pipe we obtain the equivalent system to (3.1) in diagonal form as

$$
\partial_{t}\left(\begin{array}{l}
R_{+} \\
R_{-}
\end{array}\right)+D\left(R_{+}, R_{-}\right) \partial_{x}\left(\begin{array}{c}
R_{+} \\
R_{-}
\end{array}\right)=G\left(R_{+}, R_{-}\right) .
$$

With the notation $R=\left(R_{+}, R_{-}\right)^{T}$ we can write (3.12) as

$$
\partial_{t} R+D(R) \partial_{x} R=G(R)
$$

In this diagonal form we can study the classical solutions by using characteristic curves. For our analysis we linearize the system around a stationary state. The existence of classical stationary states is discussed in Section 2. For a subsonic stationary state $\bar{R}$, we have $\partial_{t} \bar{R}=0$ and

$$
D(\bar{R}) \partial_{x} \bar{R}=G(\bar{R})
$$

hence $\partial_{x} \bar{R}=D(\bar{R})^{-1} G(\bar{R})$. Locally around this stationary state $\bar{R}$, we can write our system states in the form $R=\bar{R}+r$. We derive a partial differential equation for $r$, starting with the left-hand side of (3.13):

$$
\begin{aligned}
\partial_{t}(r+\bar{R})+D(r+\bar{R}) \partial_{x}(r+\bar{R}) & =\partial_{t} r+D(r+\bar{R}) \partial_{x} r+D(r+\bar{R}) D(\bar{R})^{-1} G(\bar{R}) \\
& =G(r+\bar{R}),
\end{aligned}
$$

hence for $r$ we obtain the partial differential equation

$$
\partial_{t} r+D(r+\bar{R}) \partial_{x} r=G(r+\bar{R})-D(r+\bar{R}) D(\bar{R})^{-1} G(\bar{R})=: \tilde{G}(\bar{R}, r)
$$

where the source term $\tilde{G}$ has the property

$$
\tilde{G}(\bar{R}, 0)=0 .
$$


We use the notation $\tilde{G}(\bar{R}, r)=\left(\tilde{G}_{+}(\bar{R}, r), \tilde{G}_{-}(\bar{R}, r)\right)^{T}$. If $q \geq 0$ we have

$$
\begin{aligned}
\tilde{G}_{ \pm}(\bar{R}, r) & =\frac{f_{g}}{8 D}\left[\left(\bar{R}_{+}+\bar{R}_{-}+r_{+}+r_{-}\right)^{2}-\frac{\bar{\lambda}_{ \pm}-\frac{r_{+}+r_{-}}{2}}{\bar{\lambda}_{ \pm}}\left(\bar{R}_{+}+\bar{R}_{-}\right)^{2}\right] \\
& =\frac{f_{g}}{8 D}\left\{\left(r_{+}+r_{-}\right)\left[2\left(\bar{R}_{+}+\bar{R}_{-}\right)+\frac{1}{2 \bar{\lambda}_{ \pm}}\left(\bar{R}_{+}+\bar{R}_{-}\right)^{2}\right]+\left(r_{+}+r_{-}\right)^{2}\right\} \\
& =\frac{f_{g}}{8 D}\left\{-\left(r_{+}+r_{-}\right)\left[\frac{2 \frac{\bar{q}}{\bar{\rho}}\left( \pm 2 a+\frac{\bar{q}}{\bar{\rho}}\right)}{\frac{\bar{q}}{\bar{\rho}} \pm a}\right]+\left(r_{+}+r_{-}\right)^{2}\right\} .
\end{aligned}
$$

Hence if $\left|r_{+}+r_{-}\right|$is small enough we have $\left(r_{+}+r_{-}\right) \tilde{G}_{ \pm}(\bar{R}, r)<0$. In other words, if $\left|r_{+}+r_{-}\right|$is small enough we have

$$
\operatorname{sign} \tilde{G}_{ \pm}(\bar{R}, r)=-\operatorname{sign}\left(r_{+}+r_{-}\right) .
$$

We have $\left|\tilde{G}_{ \pm}(\bar{R}, r)\right|=O\left(\left|r_{+}+r_{-}\right|\right)$, in particular $r_{+}+r_{-}=0$ implies $\tilde{G}_{ \pm}(\bar{R}, r)=0$.

\subsection{Scaling of the state variables by a factor $\theta>0$}

An essential observation for our analysis is the fact that if $\left(\rho^{(i)}, q^{(i)}\right)$ is a solution to the system (1.1) for given control function $u$, then for any $\theta>0$ also $\left(\theta \rho^{(i)}, \theta q^{(i)}\right)$ is a solution with the control function $\theta u$.

For $\theta>0$ sufficiently small in this way we obtain initial data and a control function with arbitrarily small $C^{1}$-norm. It is important that this scaling does not change the subsonic nature of the state. In terms of the Riemann invariants this scaling corresponds to replacing $\left(R_{+}^{(i)}, R_{-}^{(i)}\right)$ by

$$
R_{+, \theta}^{(i)}=R_{+}^{(i)}-a \ln (\theta), R_{-, \theta}^{(i)}=R_{-}^{(i)}+a \ln (\theta),
$$

hence $R_{+}^{(i)}+R_{-}^{(i)}=R_{+, \theta}^{(i)}+R_{-, \theta}^{(i)}$. Interestingly, in terms of Riemann invariants the scaling does not lead to a change in the maximum norms of the derivatives. Moreover, it also does not change the eigenvalues of the system. However, for the control function the corresponding scaling remains $\theta u(t)$. Note that if (3.6) holds then also $R_{-, \theta}^{(2)}+R_{+, \theta}^{(1)} \leq 0$ for all $\theta>0$. For all $\theta>0$ equations (3.3) and (3.2) imply

$$
\theta q^{*}=Q\left(R_{+, \theta}, F_{-}\left(\theta q^{*}, R_{+, \theta}\right)\right)=Q\left(R_{+, \theta}, F_{-}\left(q^{*}, R_{+}\right)+a \ln (\theta)\right) .
$$

Hence (3.3) implies $F_{-}\left(\theta q^{*}, R_{+, \theta}\right)=F_{-}\left(q^{*}, R_{+}\right)+a \ln (\theta)$. Analogously, we obtain the equation $F_{+}\left(\theta q^{*}, R_{-, \theta}\right)=$ $F_{+}\left(q^{*}, R_{-}\right)-a \ln (\theta)$.

Since the physical variables corresponding to the scaled Riemann invariants $R_{\theta}$ with the control $\theta u(t)$ satisfy the compressor equations (1.5a), (1.5b) if and only if they are satisfied for $\theta=1$, the compressor equations for $R_{\theta}$ have the following form:

$$
\left(\begin{array}{c}
R_{-, \theta}^{(1)}\left(t, L_{1}\right) \\
R_{+, \theta}^{(2)}(t, 0)
\end{array}\right)=\left(\begin{array}{c}
\Psi_{1}\left(R_{+, \theta}^{(1)}\left(t, L_{1}\right), R_{-, \theta}^{(2)}(t, 0), \theta u(t)\right) \\
\Psi_{2}\left(R_{+, \theta}^{(1)}\left(t, L_{1}\right), R_{-, \theta}^{(2)}(t, 0), \theta u(t)\right)
\end{array}\right) .
$$

Note that (3.17) holds for $\theta=1$ if and only if it holds for all $\theta>0$.

\section{Existence of SOLUTiOns}

The results presented below give the existence of solutions $R_{ \pm}^{(i)}=\bar{R}_{ \pm}^{(i)}+r_{ \pm}^{(i)}$ for a a fixed given time $T>0$, a given subsonic stationary state $\bar{R}_{ \pm}^{(i)}$ and a given control $u=\bar{u}+v(t)$. The local result refers to the case 
where $\bar{u} / \bar{q}$ is bounded and $r_{ \pm}^{(i)}(0, x)$ and $v(t)$ are sufficiently small in the $C^{1}$-norm. For the global result these assumptions are replaced by the assertion that $\|v\|_{C^{1}} / \bar{q}$ is sufficiently small.

\subsection{Local existence result}

In this section we apply a result of Wang [35] about semi-global $C^{1}$-solutions for initial-boundary value problems for quasilinear hyperbolic systems, see also [21-26]. For the system introduced in Section 1, we obtain the existence of $C^{1}$-solutions in a $C^{1}$-neighbourhood of a stationary subsonic $C^{1}$-state $\bar{R}$.

Theorem 4.1. Let $\bar{q}>0$ and $\bar{\rho}_{0}>0$ be given such that $\bar{q}<a \bar{\rho}_{0}$. Assume that $\bar{q}$ and $\bar{\rho}_{0}$ define a subsonic stationary $C^{1}$-state with the constant flow rate $\bar{q}$ and constant boundary density $\rho^{(1)}(t, 0)=\bar{\rho}_{0}$ that satisfies the isothermal Euler equations along the pipes and the coupling conditions (1.5a), (1.5b) with the constant control $\bar{u} \geq 0$ in the compressor. Assume that

$$
\bar{u} \in\left[0, \bar{q}\left\{\left[\exp \left(\frac{\bar{q}}{a \rho^{(1)}\left(L_{1}\right)}\right)\right]^{\kappa}-1\right\}\right)
$$

where $\rho^{(1)}\left(L_{1}\right)$ denotes the constant density for the stationary state at the end of the first pipe.

Denote the corresponding Riemann invariants by $\bar{R}=\left(\bar{R}^{(1)}, \bar{R}^{(2)}\right)$ and let $\tilde{\rho}_{1}=\rho^{(2)}\left(L_{2}\right)$ denote the constant density for the stationary state at the end of the second pipe.

Let $T>0$ be given. Then there exists $\varepsilon>0$ such that the following statement holds true:

Let initial data $\bar{R}^{(1)}(x)+b^{(1)}(x), \bar{R}^{(2)}(x)+b^{(2)}(x)$ with $\left\|b^{(1)}\right\|_{C^{1}\left(\left[0, L_{1}\right]\right)} \leq \varepsilon,\left\|b^{(2)}\right\|_{C^{1}\left(\left[0, L_{2}\right]\right)} \leq \varepsilon$ and a control function $u(t)=\bar{u}+v(t) \geq 0$ with $\|v\|_{C^{1}([0, T])} \leq \varepsilon$ be given such that the $C^{1}$-compatibility conditions are satisfied at the boundary points $x=0$ of the first pipe and $x=L_{2}$ of the second pipe with the physical boundary data

$$
\begin{aligned}
\rho^{(1)}(t, 0) & =\bar{\rho}_{0}, \\
\rho^{(2)}\left(t, L_{2}\right) & =\tilde{\rho}_{1}
\end{aligned}
$$

and such that $u(t)$ satisfies the $C^{1}$-compatibility conditions at the compressor.

Then, there exists a $C^{1}$-function $\left(r^{(1)}, r^{(2)}\right) \in\left(C^{1}\left([0, T] \times\left[0, L_{1}\right]\right)\right)^{2} \times\left(C^{1}\left([0, T] \times\left[0, L_{2}\right]\right)\right)^{2}$ such that $\bar{R}+r$ is a solution of the system (1.4a), (1.4b) in terms of Riemann invariants.

More precisely, for $i \in\{1,2\}$ the function $r^{(i)}$ solves the partial differential equations on $\left[0, L_{i}\right]$

$$
\partial_{t} r^{(i)}+D\left(r^{(i)}+\bar{R}^{(i)}\right) \partial_{x} r^{(i)}=G\left(r^{(i)}+\bar{R}^{(i)}\right)-D\left(r^{(i)}+\bar{R}^{(i)}\right) D\left(\bar{R}^{(i)}\right)^{-1} G\left(\bar{R}^{(i)}\right)=: \tilde{G}^{(i)}\left(\bar{R}^{(i)}, r^{(i)}\right)
$$

and satisfies the initial conditions $r^{(i)}(0, x)=\bar{R}^{(i)}(x)+b^{(i)}(x)$ on $\left[0, L_{i}\right]$, the boundary conditions

$$
\begin{aligned}
r_{+}^{(1)}(t, 0) & =r_{-}^{(1)}(t, 0), \\
r_{-}^{(2)}\left(t, L_{2}\right) & =r_{+}^{(2)}\left(t, L_{2}\right)
\end{aligned}
$$

and the coupling conditions

$$
\left(\begin{array}{c}
r_{-}^{(1)}\left(t, L_{1}\right) \\
r_{+}^{(2)}(t, 0)
\end{array}\right)=\left(\begin{array}{c}
\Psi_{1}\left(r_{+}^{(1)}\left(t, L_{1}\right)+\bar{R}_{+}^{(1)}\left(L_{1}\right), r_{-}^{(2)}(t, 0)+\bar{R}_{-}^{(2)}(0), u(t)\right)-\bar{R}_{-}^{(1)}\left(L_{1}\right) \\
\Psi_{2}\left(r_{+}^{(1)}\left(t, L_{1}\right)+\bar{R}_{+}^{(1)}\left(L_{1}\right), r_{-}^{(2)}(t, 0)+\bar{R}_{-}^{(2)}(0), u(t)\right)-\bar{R}_{+}^{(2)}(0)
\end{array}\right) .
$$

Proof. Without restriction, assume that our pipes have equal length that is $L_{1}=L_{2}=L$. This can be achieved by a parameter change $x \mapsto x L / L_{i}$ for $i \in\{1,2\}$. As a consequence of this change, the corresponding eigenvalues are multiplied by factors $L / L_{i}$. The Riemann invariants are not affected by this parameter change (see [20]). 
The coupling conditions become boundary conditions at the end $L$ if we transform the variable $x$ corresponding to the second pipe by $x \mapsto L-x$ which leads to a change in sign of both eigenvalues for the second pipe.

We want to apply Theorem 2.1 from [35] about the existence of a semi-global $C^{1}$-solution on the time interval $[0, T]$. For this purpose, we write our partial differential equation as a hyperbolic system for $r$ in diagonal form with a $4 \times 4$ diagonal matrix containing the eigenvalues and the vector of four unknown functions

$$
\left(r_{-}^{(1)}, r_{+}^{(2)}, r_{+}^{(1)}, r_{-}^{(2)}\right)^{T}
$$

In fact, in our case the in the notation from [35] (1.1) the vectors $l_{i}$ are canonical unit vectors and $a_{i}=b_{i}=$ $c_{i}=0$. For the source terms (3.15) implies the equations $\tilde{G}^{(i)}\left(\bar{R}^{(i)}, r^{(i)}\right)=0(i \in\{1,2\})$ which guarantee that equation (1.4) required in [35] holds.

The transformation of the physical boundary conditions (4.2), (4.3) with constant density to the boundary conditions in Riemann invariants (4.4a), (4.4b) is given in Section 3.3. Moreover, we can write our coupling conditions in the form (4.5) (where as mentioned before the end zero of the second pipe is transformed to $x=0$ ), since our assumption (4.1) implies that $u(t)$ satisfies the assumption (3.9) of Lemma 3.1 if $\varepsilon$ is sufficiently small, which in turn implies that we can write the coupling conditions in the form (3.8).

Hence we have a system with boundary conditions of the form (1.10), (1.11) from [35] with $H_{1}(t)=$ $\Psi_{1}\left(\bar{R}_{+}^{(1)}\left(L_{1}\right), \bar{R}_{-}^{(2)}(0), u(t)\right)-\bar{R}_{-}^{(1)}\left(L_{1}\right), H_{2}(t)=\Psi_{2}\left(\bar{R}_{+}^{(1)}\left(L_{1}\right), \bar{R}_{-}^{(2)}(0), u(t)\right)-\bar{R}_{+}^{(2)}(0)$ and $\|u\|_{C^{1}([0, T])}$ small implies $\left\|H_{i}\right\|_{C^{1}([0, T])}(i \in\{1,2\})$ small and such that (1.13) also holds. Therefore Theorem 2.1 from [35] implies the assertion.

\subsection{Global existence result}

Due to the scaling properties of the system described in Section 3.5 our local existence result implies a global existence result for $C^{1}$-compatible control functions. To obtain the global result, we combine the observation that for all solutions of our system we can obtain scaled solutions with $\theta>0$ as described in Section 3.5 with the local result from Theorem 4.1 to obtain the following lemma:

Lemma 4.2. Let $\theta>0$ be given. Let $\bar{q}>0$ and $\bar{\rho}_{0}>0$ be given such that $\bar{q}<a \bar{\rho}_{0}$. Assume that $\theta \bar{q}$ and $\theta \bar{\rho}_{0}$ define a subsonic stationary $C^{1}$-state with the constant flow rate $\theta \bar{q}$ and constant boundary density $\rho^{(1)}(t, 0)=\theta \bar{\rho}_{0}$ that satisfies the isothermal Euler equations along the pipes and the coupling conditions (1.5a), (1.5b) with the constant control $\theta \bar{u} \geq 0$ in the compressor. Assume that (4.1) holds where $\rho^{(1)}\left(L_{1}\right)$ denotes the constant density for the stationary state with $\theta=1$ at the end of the first pipe.

Denote the corresponding Riemann invariants by $\bar{R}_{\theta}=\left(\bar{R}_{\theta}^{(1)}, \bar{R}_{\theta}^{(2)}\right)$ and let $\tilde{\rho}_{1}=\rho^{(2)}\left(L_{2}\right)$ denote the constant density for $\theta=1$ for the stationary state at the end of the second pipe.

Let $T>0$ be given. Then there exists $\varepsilon>0$ such that the following statement holds uniformly for all $\theta>0$ :

For all initial data $\bar{R}_{\theta}^{(1)}(x)+b^{(1)}(x), \bar{R}_{\theta}^{(2)}(x)+b^{(2)}(x)$ with $\left\|b^{(1)}\right\|_{C^{1}\left(\left[0, L_{1}\right]\right)} \leq \varepsilon,\left\|b^{(2)}\right\|_{C^{1}\left(\left[0, L_{2}\right]\right)} \leq \varepsilon$ and all control functions $u(t)=\theta \bar{u}+v(t) \geq 0$ with $\|v\|_{C^{1}([0, T])} \leq \theta \varepsilon$ such that the $C^{1}$-compatibility conditions are satisfied at the boundary points $x=0$ of the first pipe and $x=L_{2}$ of the second pipe with the physical boundary data

$$
\begin{aligned}
\rho^{(1)}(t, 0) & =\theta \bar{\rho}_{0}, \\
\rho^{(2)}\left(t, L_{2}\right) & =\theta \tilde{\rho}_{1}
\end{aligned}
$$

and such that $u(t)$ satisfies the $C^{1}$-compatibility conditions on the compressor there exists a $C^{1}$-function $\left(r^{(1)}\right.$, $\left.r^{(2)}\right) \in\left(C^{1}\left([0, T] \times\left[0, L_{1}\right]\right)\right)^{2} \times\left(C^{1}\left([0, T] \times\left[0, L_{2}\right]\right)\right)^{2}$ such that $\bar{R}_{\theta}+r$ is a solution of our system in terms of Riemann invariants. More precisely, for $i \in\{1,2\}$ the function $r^{(i)}$ solves on $\left[0, L_{i}\right]$ the system

$$
\partial_{t} r^{(i)}+D\left(r^{(i)}+\bar{R}_{\theta}^{(i)}\right) \partial_{x} r^{(i)}=G\left(r^{(i)}+\bar{R}_{\theta}^{(i)}\right)-D\left(r^{(i)}+\bar{R}_{\theta}^{(i)}\right) D\left(\bar{R}_{\theta}^{(i)}\right)^{-1} G\left(\bar{R}_{\theta}^{(i)}\right)=: \tilde{G}^{(i)}\left(\bar{R}^{(i)}, r^{(i)}\right)
$$


and satisfies the initial conditions $r^{(i)}(0, x)=\bar{R}_{\theta}^{(i)}(x)+b^{(i)}(x)$ on $\left[0, L_{i}\right]$ and the boundary conditions

$$
\begin{aligned}
r_{+}^{(1)}(t, 0) & =r_{-}^{(1)}(t, 0), \\
r_{-}^{(2)}\left(t, L_{2}\right) & =r_{+}^{(2)}\left(t, L_{2}\right)
\end{aligned}
$$

and the coupling conditions

$$
\left(\begin{array}{c}
r_{-}^{(1)}\left(t, L_{1}\right) \\
r_{+}^{(2)}(t, 0)
\end{array}\right)=\left(\begin{array}{c}
\Psi_{1}\left(r_{+}^{(1)}\left(t, L_{1}\right)+\bar{R}_{+, \theta}^{(1)}\left(L_{1}\right), r_{-}^{(2)}(t, 0)+\bar{R}_{-, \theta}^{(2)}(0), u(t)\right)-\bar{R}_{-, \theta}^{(1)}\left(L_{1}\right) \\
\Psi_{2}\left(r_{+}^{(1)}\left(t, L_{1}\right)+\bar{R}_{+, \theta}^{(1)}\left(L_{1}\right), r_{-}^{(2)}(t, 0)+\bar{R}_{-, \theta}^{(2)}(0), u(t)\right)-\bar{R}_{+, \theta}^{(2)}(0)
\end{array}\right) .
$$

Now we give a result that applies if the compressor initially is switched off. It states that starting with a subsonic stationary state with positive flow rate $\bar{q}>0$ and $\bar{u}=0$ we obtain the existence of $C^{1}$-solutions for all $C^{1}$-control functions $u(t)$ that satisfy the $C^{1}$-compatibility conditions and for which $u(t) / \bar{q}$ is sufficiently small.

Theorem 4.3. Let $T>0$ and $\gamma>0$ be given. There exists $\varepsilon>0$ such that for all subsonic stationary initial $C^{1}$-states with constant flow rate $\bar{q}>0$ and switched off compressor (that is control value zero at the compressor) and boundary density $\rho^{(1)}(t, 0)=\gamma \bar{q}$ at $x=0$ in the first pipe and all for control functions $u(t)$ satisfying the $C^{1}$-compatibility conditions at the compressor and $\|u\|_{C^{1}([0, T])} / \bar{q} \leq \varepsilon$, there exists a $C^{1}$-solution on the time interval $[0, T]$ with constant boundary densities.

Proof. Fix a flow rate $q_{1}>0$. Let $\rho_{1}>0$ denote a constant boundary density at $x=0$ in the first pipe that generates a stationary subsonic initial state with the flow rate $q_{1}>0$. In Lemma 4.2 applied to the case with this stationary subsonic initial state we have $\bar{u}=0$ and hence (4.1) holds and $v(t)=u(t)$. Moreover, due to the assumption that the initial state is stationary we have $b^{(1)}=b^{(2)}=0$. We choose $\varepsilon>0$ from Lemma 4.2 . Lemma 4.2 states that uniformly for all $\theta>0$ for the stationary initial states $\bar{R}_{\theta}$ corresponding to the physical variables $\theta q_{1}, \theta \rho_{1}$ and control functions $v$ at the compressor that satisfy the $C^{1}$-compatibility conditions and for which

$$
\|v\|_{C^{1}([0, T])} \leq \theta \varepsilon
$$

a $C^{1}$-state is generated. Inequality (4.10) is satisfied if $\frac{\|v\|_{C^{1}([0, T])}}{\theta q_{1}} \leq \frac{\varepsilon}{q_{1}}$. We obtain the last inequality in the statement of Theorem 4.3 by replacing $\theta q_{1}$ by $\bar{q}$ and by letting $\frac{\varepsilon}{q_{1}}$ be the $\varepsilon$ in the statement of Theorem 4.3 . Define $\gamma=\rho_{1} / q_{1}$. Then we have $\theta \rho_{1}=\theta \gamma q_{1}=\gamma \bar{q}$.

\section{Stabilization}

In this section we consider the problem to stabilize our system locally around a given classical stationary solution using a boundary feedback and a feedback law at the compressor. Note that in the literature about stabilization of hyperbolic systems, usually systems without source terms are considered (see [7,20]), whereas in the system that we consider here the influence of the source term is essential. We use a Lyapunov function taking care of both the special source term and of the fact that the coefficients in the transport equation are space dependent, see below. Then, the idea to stabilize the system is to use a Riemann control law at the two boundary points and a control that yields the correct flow rate at the compressor. For our analysis it is important that the source term satisfies (3.16).

In order to highlight the idea of the particular choice of the Lyapunov function we consider at first the toy example on $[0,1] \times(0, \infty)$

$$
\partial_{t} a+c(x) \partial_{x} a=d(x, a)
$$

with $c(x)>0$ and $a(t, 0)=a_{0}=k a(t, 1)$. A coupled system of these equations comprises a linearized gas model and will be studied below. For the given equation we extend recently introduced Lyapunov functions $[8,9,11,13]$ 
and use the following ansatz

$$
E(t)=\int_{0}^{1} \exp \left(-\mu \int_{0}^{x} \frac{1}{c(s)} \mathrm{d} s\right) a^{2} \frac{A}{c(x)} \mathrm{d} x .
$$

Using careful estimates on the source term $d(x, a)$ we will establish for the linearized gas model that

$$
E^{\prime}(t) \leq-\mu E(t)+\text { boundary terms. }
$$

This idea is extended to the nonlinear system (that is $c=c(x, a)$ ) and using appropriate boundary conditions at the end of the pipe and a suitable compressor control, we prove exponential decay in the $L^{2}$-norm in Theorem 5.3.

\subsection{A single pipe: Stabilization of the linearized system}

To construct a stabilizing feedback law, we consider the following linear approximation of the characteristic form (3.14):

$$
\begin{aligned}
& \partial_{t} r_{+}+\bar{\lambda}_{+} \partial_{x} r_{+}=-K_{+}\left(r_{+}+r_{-}\right), \\
& \partial_{t} r_{-}+\bar{\lambda}_{-} \partial_{x} r_{-}=-K_{-}\left(r_{+}+r_{-}\right)
\end{aligned}
$$

with the appropriate functions $K_{+}(x)>0, K_{-}(x)>0$ from (3.14) that is

$$
K_{ \pm}=\frac{f_{g}}{8 D}\left[\frac{2 \frac{\bar{q}}{\bar{\rho}}\left( \pm 2 a+\frac{\bar{q}}{\bar{\rho}}\right)}{\frac{\bar{q}}{\bar{\rho}} \pm a}\right]
$$

In the source term we have omitted the quadratic term $+\left(r_{+}+r_{-}\right)^{2}$. In this approximation the fixed eigenvalues $\bar{\lambda}_{ \pm}$and the characteristic curves corresponding to the steady state $\left(\bar{R}_{+}, \bar{R}_{-}\right)$are used.

Let $\mu>0$ be given. Define the functions

$$
\begin{aligned}
& h_{+}(x)=\exp \left(-\mu \int_{0}^{x} \frac{1}{\bar{\lambda}_{+}(s)} \mathrm{d} s\right), \\
& h_{-}(x)=\exp \left(\mu \int_{0}^{x} \frac{1}{-\bar{\lambda}_{-}(s)} \mathrm{d} s\right) .
\end{aligned}
$$

Then for all $x \in[0, L]$ we have

$$
\exp \left(\mu\left(\int_{0}^{L}-\frac{1}{\bar{\lambda}_{+}}+\frac{1}{\bar{\lambda}_{-}}\right)\right)=\frac{h_{+}(L)}{h_{-}(L)} \leq \frac{h_{+}(x)}{h_{-}(x)} \leq \frac{h_{+}(0)}{h_{-}(0)}=1
$$

and $h_{+}(x) \in\left[h_{+}(L), 1\right], h_{-}(x) \in\left[1, h_{-}(L)\right]$.

For $A>0, B>0$ define the Lyapunov function

$$
E(t)=\int_{0}^{L} \frac{A}{\bar{\lambda}_{+}(x)} r_{+}^{2}(t, x) h_{+}(x)+\frac{B}{-\bar{\lambda}_{-}(x)} r_{-}^{2}(t, x) h_{-}(x) \mathrm{d} x .
$$

Definition (5.3) is a generalization of the definition in [7] to the case where the eigenvalues depend on the spatial variable. This is essential for our problem, since the stationary states are non-constant in space. 
The time derivative of $E$ is

$$
\begin{aligned}
E^{\prime}(t)= & \int_{0}^{L}-A h_{+}(x) \partial_{x}\left[r_{+}(t, x)\right]^{2}-2 A h_{+}(x) \frac{K_{+}(x)}{\bar{\lambda}_{+}} r_{+} *\left(r_{+}+r_{-}\right) \mathrm{d} x \\
& +\int_{0}^{L} B h_{-}(x) \partial_{x}\left[r_{-}(t, x)\right]^{2}+2 B h_{-}(x) \frac{K_{-}(x)}{\bar{\lambda}_{-}} r_{-} *\left(r_{+}+r_{-}\right) \mathrm{d} x \\
= & {\left.\left[-A h_{+}(x)\left[r_{+}(t, x)\right]^{2}+B h_{-}(x)\left[r_{-}(t, x)\right]^{2}\right]\right|_{x=0} ^{L} } \\
& -\int_{0}^{L} A \frac{\mu}{\bar{\lambda}_{+}} h_{+}(x)\left[r_{+}(t, x)\right]^{2}-B \frac{\mu}{\bar{\lambda}_{-}} h_{-}(x)\left[r_{-}(t, x)\right]^{2} \mathrm{~d} x \\
& -\int_{0}^{L} 2\left[A h_{+}(x) \frac{K_{+}(x)}{\bar{\lambda}_{+}} r_{+}+B h_{-}(x) \frac{K_{-}(x)}{-\bar{\lambda}_{-}} r_{-}\right]\left(r_{+}+r_{-}\right) \mathrm{d} x \\
= & \mu E(t)+\left.\left[-A h_{+}(x)\left[r_{+}(t, x)\right]^{2}+B h_{-}(x)\left[r_{-}(t, x)\right]^{2}\right]\right|_{x=0} ^{L} \\
& -\int_{0}^{L} 2 A h_{+}(x) \frac{K_{+}(x)}{\bar{\lambda}_{+}} r_{+}^{2}+2 B h_{-}(x) \frac{K_{-}(x)}{-\bar{\lambda}_{-}} r_{-}^{2} \mathrm{~d} x \\
& -\int_{0}^{L} 2 A h_{+}(x) \frac{K_{+}(x)}{\bar{\lambda}_{+}} r_{+} r_{-}+2 B h_{-}(x) \frac{K_{-}(x)}{-\bar{\lambda}_{-}} r_{+} r_{-} \mathrm{d} x .
\end{aligned}
$$

Using the inequality $\left|r_{+} r_{-}\right| \leq\left(r_{+}^{2}+r_{-}^{2}\right) / 2$ we obtain

$$
\begin{aligned}
E^{\prime}(t) \leq & -\mu E(t)+\left.\left[-A h_{+}(x)\left[r_{+}(t, x)\right]^{2}+B h_{-}(x)\left[r_{-}(t, x)\right]^{2}\right]\right|_{x=0} ^{L} \\
& -\int_{0}^{L} A h_{+}(x) \frac{K_{+}(x)}{\bar{\lambda}_{+}} r_{+}^{2}+B h_{-}(x) \frac{K_{-}(x)}{-\bar{\lambda}_{-}} r_{-}^{2} \mathrm{~d} x \\
& +\int_{0}^{L} A h_{+}(x) \frac{K_{+}(x)}{\bar{\lambda}_{+}} r_{-}^{2}+B h_{-}(x) \frac{K_{-}(x)}{-\bar{\lambda}_{-}} r_{+}^{2} \mathrm{~d} x .
\end{aligned}
$$

Case 1. Assume that

$$
\frac{A}{B} \geq \sup _{x \in[0, L]} \frac{\bar{\lambda}_{+}(x)}{-\bar{\lambda}_{-}(x)} \frac{K_{-}(x)}{K_{+}(x)} \frac{h_{-}(x)}{h_{+}(x)}
$$

Then we have the inequality

$$
-\frac{A h_{+} K_{+}}{\bar{\lambda}_{+}}+\frac{B h_{-} K_{-}}{-\bar{\lambda}_{-}} \leq 0
$$

which implies

$$
\begin{aligned}
E^{\prime}(t) \leq-\mu E(t)+\left[-A h_{+}(x)\left[r_{+}(t, x)\right]^{2}+B h_{-}(x)\left[r_{-}(t, x)\right]^{2}\right] & \left.\right|_{x=0} ^{L} \\
& +\int_{0}^{L}\left[-B h_{-}(x) \frac{K_{-}(x)}{-\bar{\lambda}_{-}}+A h_{+}(x) \frac{K_{+}(x)}{\bar{\lambda}_{+}}\right] r_{-}^{2} \mathrm{~d} x .
\end{aligned}
$$

Assume that

$$
\frac{A}{B} \leq \inf _{x \in[0, L]} \frac{\bar{\lambda}_{+}(x)}{-\bar{\lambda}_{-}(x)} \frac{K_{-}(x)}{K_{+}(x)}\left(1+\frac{\mu}{2 K_{-}(x)}\right) .
$$

Then since $h_{-}(x) / h_{+}(x) \geq 1$ we obtain the inequality

$$
-\left(\frac{\mu}{2}+K_{-}\right) \frac{B h_{-}}{-\bar{\lambda}_{-}}+\frac{A h_{+} K_{+}}{\bar{\lambda}_{+}} \leq 0
$$


which implies

$$
E^{\prime}(t) \leq-\frac{\mu}{2} E(t)+\left.\left[-A h_{+}(x)\left[r_{+}(t, x)\right]^{2}+B h_{-}(x)\left[r_{-}(t, x)\right]^{2}\right]\right|_{x=0} ^{L} .
$$

Case 2. Assume that

$$
\frac{B}{A} \geq \sup _{x \in[0, L]} \frac{-\bar{\lambda}_{-}(x)}{\bar{\lambda}_{+}(x)} \frac{K_{+}(x)}{K_{-}(x)} \frac{h_{+}(x)}{h_{-}(x)}
$$

Then we have the inequality

$$
\frac{A h_{+} K_{+}}{\bar{\lambda}_{+}}-\frac{B h_{-} K_{-}}{-\bar{\lambda}_{-}} \leq 0
$$

which implies

$$
\begin{aligned}
E^{\prime}(t) \leq & -\mu E(t)+\left.\left[-A h_{+}(x)\left[r_{+}(t, x)\right]^{2}+B h_{-}(x)\left[r_{-}(t, x)\right]^{2}\right]\right|_{x=0} ^{L} \\
& +\int_{0}^{L}\left[-A h_{+}(x) \frac{K_{+}(x)}{\bar{\lambda}_{+}}+B h_{-}(x) \frac{K_{-}(x)}{-\bar{\lambda}_{-}}\right] r_{+}^{2} \mathrm{~d} x .
\end{aligned}
$$

Assume that

$$
\frac{B}{A} \leq \inf _{x \in[0, L]} \frac{-\bar{\lambda}_{-}(x)}{\bar{\lambda}_{+}(x)} \frac{h_{+}(x)}{h_{-}(x)} \frac{K_{+}(x)}{K_{-}(x)}\left(1+\frac{\mu}{2 K_{+}(x)}\right) .
$$

Then we obtain the inequality

$$
-\left(\frac{\mu}{2}+K_{+}\right) \frac{A h_{+}}{\bar{\lambda}_{+}}+\frac{B h_{-} K_{-}}{-\bar{\lambda}_{-}} \leq 0
$$

which implies inequality (5.6).

Lemma 5.1. Consider the linearized system (5.1a), (5.1b) on the set $[0, T] \times[0, L]$. Assume that a $C^{1}$-solution exists on $[0, T] \times[0, L]$. Define

Assume that

$$
\frac{1}{\mu}=\int_{0}^{L} \frac{1}{\bar{\lambda}_{+}(x)}-\frac{1}{\bar{\lambda}_{-}(x)} \mathrm{d} x
$$

$$
\sup _{x \in[0, L]} \frac{\bar{\lambda}_{+}(x)}{-\bar{\lambda}_{-}(x)} \frac{K_{-}(x)}{K_{+}(x)} \exp (1) \leq \inf _{x \in[0, L]} \frac{\bar{\lambda}_{+}(x)}{-\bar{\lambda}_{-}(x)} \frac{K_{-}(x)}{K_{+}(x)}\left(1+\frac{\mu}{2 K_{-}(x)}\right)
$$

or

$$
\sup _{x \in[0, L]} \frac{-\bar{\lambda}_{-}(x)}{\bar{\lambda}_{+}(x)} \frac{K_{+}(x)}{K_{-}(x)} \exp (1) \leq \inf _{x \in[0, L]} \frac{-\bar{\lambda}_{-}(x)}{\bar{\lambda}_{+}(x)} \frac{K_{+}(x)}{K_{-}(x)}\left(1+\frac{\mu}{2 K_{+}(x)}\right) .
$$

Then there exist real numbers $A>0$ and $B>0$ such that [(5.4) and (5.5)] or [(5.7) and (5.8)] hold and

$$
E^{\prime}(t) \leq-\frac{\mu}{2} E(t)+\left.\left[-A h_{+}(x)\left[r_{+}(t, x)\right]^{2}+B h_{-}(x)\left[r_{-}(t, x)\right]^{2}\right]\right|_{x=0} ^{L}
$$

Remark 5.1. The inequalities (5.10) and (5.11) hold true, whenever $1 / \mu$ is sufficiently small. The constant $\mu$ is large, if the length of the pipe $L$ becomes small. This implies that stabilization becomes 'easier' as soon as the pipe length decreases.

Proof. Case 1. If (5.4) and (5.5) hold true, (5.6) implies the desired inequality. It remains to show that (5.10) implies that we can find $A / B$ such that (5.4) and (5.5) hold with $\mu$ defined by (5.9). Inequality (5.10) implies 
that

$$
\begin{aligned}
\sup _{x \in[0, L]} \frac{\bar{\lambda}_{+}(x)}{-\bar{\lambda}_{-}(x)} \frac{K_{-}(x)}{K_{+}(x)} \frac{h_{-}(x)}{h_{+}(x)} & \leq \sup _{x \in[0, L]} \frac{\bar{\lambda}_{+}(x)}{-\bar{\lambda}_{-}(x)} \frac{K_{-}(x)}{K_{+}(x)} \frac{h_{-}(L)}{h_{+}(L)} \\
& =\exp (1) \sup _{x \in[0, L]} \frac{\bar{\lambda}_{+}(x)}{-\bar{\lambda}_{-}(x)} \frac{K_{-}(x)}{K_{+}(x)} \\
& \leq \inf _{x \in[0, L]} \frac{\bar{\lambda}_{+}(x)}{-\bar{\lambda}_{-}(x)} \frac{K_{-}(x)}{K_{+}(x)}\left(1+\frac{\mu}{2 K_{-}(x)}\right)
\end{aligned}
$$

Thus the interval

$$
I=\left[\sup _{x \in[0, L]} \frac{\bar{\lambda}_{+}(x)}{-\bar{\lambda}_{-}(x)} \frac{K_{-}(x)}{K_{+}(x)} \frac{h_{-}(x)}{h_{+}(x)}, \inf _{x \in[0, L]} \frac{\bar{\lambda}_{+}(x)}{-\bar{\lambda}_{-}(x)} \frac{K_{-}(x)}{K_{+}(x)}\left(1+\frac{\mu}{2 K_{-}(x)}\right)\right]
$$

is nonempty and for all $A / B \in I$ the inequalities (5.4) and (5.5) hold for the particular choice of $\mu$.

Case 2. If (5.7) and (5.8) hold true, (5.6) implies the desired inequality. It remains to show that (5.11) implies that we can find $B / A$ such that (5.7) and (5.8) hold with $\mu$ defined by (5.9). Since $h_{+}(x) / h_{-}(x) \geq \exp (-1)$, Inequality (5.11) implies that

$$
\begin{aligned}
\sup _{x \in[0, L]} \frac{-\bar{\lambda}_{-}(x)}{\bar{\lambda}_{+}(x)} \frac{K_{+}(x)}{K_{-}(x)} \frac{h_{+}(x)}{h_{-}(x)} & \leq \sup _{x \in[0, L]} \frac{-\bar{\lambda}_{-}(x)}{\bar{\lambda}_{+}(x)} \frac{K_{+}(x)}{K_{-}(x)} \frac{h_{+}(0)}{h_{-}(0)} \\
& =\sup _{x \in[0, L]} \frac{-\bar{\lambda}_{-}(x)}{\bar{\lambda}_{+}(x)} \frac{K_{+}(x)}{K_{-}(x)} \\
& \leq \exp (-1) \inf _{x \in[0, L]} \frac{-\bar{\lambda}_{-}(x)}{\bar{\lambda}_{-}(x)} \frac{K_{+}(x)}{K_{-}(x)}\left(1+\frac{\mu}{2 K_{+}(x)}\right) \\
& \leq \inf _{x \in[0, L]} \frac{-\bar{\lambda}_{-}(x)}{\bar{\lambda}_{-}(x)} \frac{h_{+}(x)}{h_{-}(x)} \frac{K_{+}(x)}{K_{-}(x)}\left(1+\frac{\mu}{2 K_{+}(x)}\right) .
\end{aligned}
$$

Thus the interval

$$
I=\left[\sup _{x \in[0, L]} \frac{-\bar{\lambda}_{-}(x)}{\bar{\lambda}_{+}(x)} \frac{K_{+}(x)}{K_{-}(x)} \frac{h_{+}(x)}{h_{-}(x)}, \inf _{x \in[0, L]} \frac{-\bar{\lambda}_{-}(x)}{\bar{\lambda}_{+}(x)} \frac{h_{+}(x)}{h_{-}(x)} \frac{K_{+}(x)}{K_{-}(x)}\left(1+\frac{\mu}{2 K_{+}(x)}\right)\right]
$$

is nonempty and for all $B / A \in I$ the inequalities (5.7) and (5.8) hold for the particular choice (5.9) of $\mu$.

\subsection{A single pipe: Stabilization of the nonlinear system}

In this section we analyze feedback control for the nonlinear system, with the state dependent eigenvalues $\lambda_{ \pm}=\bar{\lambda}_{ \pm}-\frac{r_{+}+r_{-}}{2}$. We write the system (3.14) in the following form:

$$
\partial_{t} r+D(\bar{R}) \partial_{x} r=\tilde{G}(\bar{R}, r)+\frac{r_{+}+r_{-}}{2} \partial_{x} r .
$$

For the components $r_{+}, r_{-}$this yields the equations

$$
\begin{aligned}
& \partial_{t} r_{+}+\bar{\lambda}_{+} \partial_{x} r_{+}=-\tilde{K}_{+}\left(r_{+}+r_{-}\right), \\
& \partial_{t} r_{-}+\bar{\lambda}_{-} \partial_{x} r_{-}=-\tilde{K}_{-}\left(r_{+}+r_{-}\right)
\end{aligned}
$$


with the functions $\tilde{K}_{+}, \tilde{K}_{-}$defined as

$$
\tilde{K}_{ \pm}(t, x)=K_{ \pm}(x)-\frac{f_{g}}{8 D}\left(r_{+}(t, x)+r_{-}(t, x)\right)-\frac{1}{2} \partial_{x} r_{ \pm}(t, x) .
$$

Consider the Lyapunov function $E(t)$ defined as in (5.3) but with $\left(r_{+}, r_{-}\right)$denoting the solution of the nonlinear system (5.12), (5.13). This yields the time derivative

$$
\begin{aligned}
E^{\prime}(t)= & -\mu E(t)+\left.\left[-A h_{+}(x)\left[r_{+}(t, x)\right]^{2}+B h_{-}(x)\left[r_{-}(t, x)\right]^{2}\right]\right|_{x=0} ^{L} \\
& -\int_{0}^{L} 2 A h_{+} \frac{\tilde{K}_{+}}{\bar{\lambda}_{+}} r_{+}^{2}+2 B h_{-} \frac{\tilde{K}_{-}}{-\bar{\lambda}_{-}} r_{-}^{2} \mathrm{~d} x-\int_{0}^{L} 2 A h_{+} \frac{\tilde{K}_{+}}{\bar{\lambda}_{+}} r_{+} r_{-}+2 B h_{-} \frac{\tilde{K}_{-}}{-\bar{\lambda}_{-}} r_{+} r_{-} \mathrm{d} x .
\end{aligned}
$$

Lemma 5.2. Consider the nonlinear system (5.12), (5.13) on the set $[0, T] \times[0, L]$. Assume that a $C^{1}$-solution $\left(r_{+}, r_{-}\right)$exists on $[0, T] \times[0, L]$ that satisfies the inequality

$$
\frac{f_{g}}{8 D}\left(r_{+}+r_{-}\right)+\frac{1}{2} \partial_{x} r_{ \pm}<K_{ \pm}
$$

on $[0, T] \times[0, L]$. Define the number $\frac{1}{\mu}=\int_{0}^{L} \frac{1}{\lambda_{+}(x)}-\frac{1}{\lambda_{-}(x)} \mathrm{d} x$. Assume that

$$
\exp (1) \sup _{(t, x) \in[0, T] \times[0, L]} \frac{\bar{\lambda}_{+}(x)}{-\bar{\lambda}_{-}(x)} \frac{\tilde{K}_{-}(t, x)}{\tilde{K}_{+}(t, x)} \leq \inf _{(t, x) \in[0, T] \times[0, L]} \frac{\bar{\lambda}_{+}(x)}{-\bar{\lambda}_{-}(x)} \frac{\tilde{K}_{-}(t, x)}{\tilde{K}_{+}(t, x)}\left(1+\frac{\mu}{2 \tilde{K}_{-}(t, x)}\right)
$$

or

$$
\exp (1) \sup _{(t, x) \in[0, T] \times[0, L]} \frac{-\bar{\lambda}_{-}(x)}{\bar{\lambda}_{+}(x)} \frac{\tilde{K}_{+}(t, x)}{\tilde{K}_{-}(t, x)} \leq \inf _{(t, x) \in[0, T] \times[0, L]} \frac{-\bar{\lambda}_{-}(x)}{\bar{\lambda}_{+}(x)} \frac{\tilde{K}_{+}(t, x)}{\tilde{K}_{-}(t, x)}\left(1+\frac{\mu}{2 \tilde{K}_{+}(t, x)}\right)
$$

which is the case if $1 / \mu$ is sufficiently small. Then we can choose $A>0$ and $B>0$ such that [(5.4) and (5.5)] or [(5.7) and (5.8)] hold with $K$ replaced by $\tilde{K}$ defined in (5.14) and we have the inequality

$$
E^{\prime}(t) \leq-\frac{\mu}{2} E(t)+\left.\left[-A h_{+}(x)\left[r_{+}(t, x)\right]^{2}+B h_{-}(x)\left[r_{-}(t, x)\right]^{2}\right]\right|_{x=0} ^{L} .
$$

Proof. Our assumption (5.15) implies that $\tilde{K}_{ \pm}(t, x)>0$ on $[0, T] \times[0, L]$. Hence the assertion follows as in the proof of Lemma 5.1.

\subsection{Stabilization of a network with one compressor station}

In this section we propose a feedback law for the compressor that yields exponential decay of a network Lyapunov function in the $L^{2}$-sense if it is combined with appropriate boundary feedback laws at the two boundary nodes of the network, for example absorbing boundary conditions. We choose the compressor control in such a way that at the compressor the flow rate has the equilibrium value corresponding to the stationary state.

In this section we assume that the compressor is at the end $L_{1}$ of the first pipe that correspond to the interval $\left[0, L_{1}\right]$ and that the compressor is at the end 0 of the second pipe corresponding to the interval $\left[0, L_{2}\right]$. We further assume a stationary subsonic state $\bar{R}_{ \pm}^{(i)}$ to be given. Note that this stationary state has a constant mass flux through pipes and compressor due to the coupling conditions that is given by the constant

$$
\bar{q}=-\frac{\bar{R}_{+}^{(1)}+\bar{R}_{-}^{(1)}}{2} \exp \left(\frac{\bar{R}_{-}^{(1)}-\bar{R}_{+}^{(1)}}{2 a}\right)=-\frac{\bar{R}_{+}^{(2)}+\bar{R}_{-}^{(2)}}{2} \exp \left(\frac{\bar{R}_{-}^{(2)}-\bar{R}_{+}^{(2)}}{2 a}\right) .
$$


Let real numbers $A^{(1)}, B^{(1)}, A^{(2)}, B^{(2)}, \mu^{(1)}, \mu^{(2)} \in(0, \infty)$ be given. For $i \in\{1,2\}$ define the functions

$$
\begin{aligned}
& h_{+}^{(i)}(x)=\exp \left(-\mu^{(i)} \int_{0}^{x} \frac{1}{\bar{\lambda}_{+}^{(i)}(s)} \mathrm{d} s\right), \\
& h_{-}^{(i)}(x)=\exp \left(\mu^{(i)} \int_{0}^{x} \frac{1}{-\bar{\lambda}_{-}^{(i)}(s)} \mathrm{d} s\right) .
\end{aligned}
$$

Define the Lyapunov functions for the pipes

$$
\begin{aligned}
& E^{(1)}(t)=\int_{0}^{L_{1}} \frac{A^{(1)}}{\bar{\lambda}_{+}^{(1)}(x)}\left[r_{+}^{(1)}(t, x)\right]^{2} h_{+}^{(1)}(x)+\frac{B^{(1)}}{-\bar{\lambda}_{-}^{(1)}(x)}\left[r_{-}^{(1)}(t, x)\right]^{2} h_{-}^{(1)}(x) \mathrm{d} x, \\
& E^{(2)}(t)=\int_{0}^{L_{2}} \frac{A^{(2)}}{\bar{\lambda}_{+}^{(2)}(x)}\left[r_{+}^{(2)}(t, x)\right]^{2} h_{+}^{(2)}(x)+\frac{B^{(2)}}{-\bar{\lambda}_{-}^{(2)}(x)}\left[r_{-}^{(2)}(t, x)\right]^{2} h_{-}^{(2)}(x) \mathrm{d} x,
\end{aligned}
$$

and the network Lyapunov function

$$
E_{N}(t)=E^{(1)}(t)+E^{(2)}(t) .
$$

At the boundary node $x=0$ of the first pipe we assume that we have a linear feedback law

$$
r_{+}^{(1)}(t, 0)=k^{(1)} r_{-}^{(1)}(t, 0)
$$

with a constant $k^{(1)}$ such that the following inequality holds:

$$
A^{(1)}\left[r_{+}^{(1)}(t, 0)\right]^{2} \leq B^{(1)}\left[r_{-}^{(1)}(t, 0)\right]^{2} .
$$

At the boundary node $x=L_{2}$ of the second pipe we assume that we have a linear feedback law

$$
r_{-}^{(2)}\left(t, L_{2}\right)=k^{(2)} r_{+}^{(2)}\left(t, L_{2}\right)
$$

with a constant $k^{(2)}$ such that the following inequality holds:

$$
B^{(2)} h_{-}^{(2)}\left(L_{2}\right)\left[r_{-}^{(2)}\left(t, L_{2}\right)\right]^{2} \leq A^{(2)} h_{+}^{(2)}\left(L_{2}\right)\left[r_{+}^{(2)}\left(t, L_{2}\right)\right]^{2} .
$$

At the compressor node the control function $u(t)$ is chosen in such a way that $q(t)=\bar{q}$, that is the compressor power maintains a steady mass flux. Hence, the control law is given by

$$
u(t)=\bar{q}\left(\left(\frac{\rho^{(2)}(t, 0)}{\rho^{(1)}\left(t, L_{1}\right)}\right)^{\kappa}-1\right) .
$$

Then the following theorem holds:

Theorem 5.3. Assume that a $C^{1}$-solution $\left(r^{(1)}, r^{(2)}\right)$ exists on the time interval $[0, T]$ in the pipeline network for the closed loop system with the feedback conditions (5.19), (5.19), (5.23) described above such that the inequalities

$$
\frac{f_{g}}{8 D}\left(r_{+}^{(i)}+r_{-}^{(i)}\right)+\frac{1}{2} \partial_{x} r_{ \pm}^{(i)}<K_{ \pm}^{(i)}
$$

hold for $i \in\{1,2\}$. For $i \in\{1,2\}$ define the numbers

$$
\frac{1}{\mu^{(i)}}=\int_{0}^{L_{i}} \frac{1}{\bar{\lambda}_{+}^{(i)}(x)}+\frac{1}{-\bar{\lambda}_{-}^{(i)}(x)} \mathrm{d} x .
$$


Assume that we have the inequalities

$$
\exp (1) \sup _{(t, x) \in[0, T] \times\left[0, L_{1}\right]} \frac{\bar{\lambda}_{+}^{(1)}(x)}{-\bar{\lambda}_{-}^{(1)}(x)} \frac{\tilde{K}_{-}^{(1)}(t, x)}{\tilde{K}_{+}^{(1)}(t, x)} \leq \inf _{(t, x) \in[0, T] \times\left[0, L_{1}\right]} \frac{\bar{\lambda}_{+}^{(1)}(x)}{-\bar{\lambda}_{-}^{(1)}(x)} \frac{\tilde{K}_{-}^{(1)}(t, x)}{\tilde{K}_{+}^{(1)}(t, x)}\left(1+\frac{\mu^{(1)}}{2 \tilde{K}_{-}^{(1)}(t, x)}\right)
$$

which is the case if the number $1 / \mu^{(1)}$ is sufficiently small.

Assume that the quotient $A^{(1)} / B^{(1)}>0$ is in the interval

$$
I^{(1)}=\left[\exp (1) \sup _{(t, x) \in[0, T] \times\left[0, L_{1}\right]} \frac{\bar{\lambda}_{+}^{(1)}(x)}{-\bar{\lambda}_{-}^{(1)}(x)} \frac{\tilde{K}_{-}^{(1)}(t, x)}{\tilde{K}_{+}^{(1)}(t, x)}, \inf _{(t, x) \in[0, T] \times\left[0, L_{1}\right]} \frac{\bar{\lambda}_{+}^{(1)}(x)}{-\bar{\lambda}_{-}^{(1)}(x)} \frac{\tilde{K}_{-}^{(1)}(t, x)}{\tilde{K}_{+}^{(1)}(t, x)}\left(1+\frac{\mu^{(1)}}{2 \tilde{K}_{-}^{(1)}(t, x)}\right)\right] .
$$

Assume that

$$
\exp (1) \sup _{(t, x) \in[0, T] \times\left[0, L_{2}\right]} \frac{-\bar{\lambda}_{-}^{(2)}(x)}{\bar{\lambda}_{+}^{(2)}(x)} \frac{\tilde{K}_{+}^{(2)}(t, x)}{\tilde{K}_{-}^{(2)}(t, x)} \leq \inf _{(t, x) \in[0, T] \times\left[0, L_{2}\right]} \frac{-\bar{\lambda}_{-}^{(2)}(x)}{\bar{\lambda}_{+}^{(2)}(x)} \frac{\tilde{K}_{+}^{(2)}(t, x)}{\tilde{K}_{-}^{(2)}(t, x)}\left(1+\frac{\mu^{(2)}}{2 \tilde{K}_{+}^{(2)}(t, x)}\right)
$$

which is the case if the number $1 / \mu^{(2)}$ is sufficiently small.

Assume that $B^{(2)} / A^{(2)}>0$ is in the interval

$I^{(2)}=\left[\sup _{(t, x) \in[0, T] \times\left[0, L_{2}\right]} \frac{-\bar{\lambda}_{-}^{(2)}(x)}{\bar{\lambda}_{+}^{(2)}(x)} \frac{\tilde{K}_{+}^{(2)}(t, x)}{\tilde{K}_{-}^{(2)}(t, x)}, \exp (-1) \inf _{(t, x) \in[0, T] \times\left[0, L_{2}\right]} \frac{-\bar{\lambda}_{-}^{(2)}(x)}{\bar{\lambda}_{+}^{(2)}(x)} \frac{\tilde{K}_{+}^{(2)}(t, x)}{\tilde{K}_{-}^{(2)}(t, x)}\left(1+\frac{\mu^{(2)}}{2 \tilde{K}_{+}^{(2)}(t, x)}\right)\right]$.

Moreover, assume that $A^{(1)} / B^{(1)}$ and $B^{(2)} / A^{(2)}$ are sufficiently large in the sense that the inequalities (5.30) and (5.33) hold which is possible if $1 / \mu^{(1)}$ and $1 / \mu^{(2)}$ are sufficiently small. (This is possible if $L_{1}$ and $L_{2}$ are sufficiently small.)

Then the following inequality is valid:

$$
E_{N}^{\prime}(t) \leq-\frac{\mu^{(1)}}{2} E^{(1)}(t)-\frac{\mu^{(2)}}{2} E^{(2)}(t) .
$$

Hence for $\alpha=\min \left\{\frac{\mu^{(1)}}{2}, \frac{\mu^{(2)}}{2}\right\}$ we have $E_{N}^{\prime}(t) \leq-\alpha E_{N}(t)$, hence

$$
E_{N}(t) \leq E_{N}(0) \exp (-\alpha t) .
$$

Proof. Inequality (5.25) implies that assumption (5.16) of Lemma 5.2 holds for the first pipe that corresponds to the interval $\left[0, L_{1}\right]$. Inequality (5.26) implies that assumption (5.17) of Lemma 5.2 holds for the second pipe corresponding to the interval $\left[0, L_{2}\right]$. Hence for the network Lyapunov function Lemma 5.2 yields the inequality

$$
\begin{aligned}
E_{N}^{\prime}(t) \leq & -\frac{\mu^{(1)}}{2} E^{(1)}(t)-\frac{\mu^{(2)}}{2} E^{(2)}(t) \\
& +B^{(1)}\left[r_{-}^{(1)}\left(t, L_{1}\right)\right]^{2} h_{-}^{(1)}\left(L_{1}\right)-A^{(1)}\left[r_{+}^{(1)}\left(t, L_{1}\right)\right]^{2} h_{+}^{(1)}\left(L_{1}\right) \\
& +A^{(2)}\left[r_{+}^{(2)}(t, 0)\right]^{2} h_{+}^{(2)}(0)-B^{(2)}\left[r_{-}^{(2)}(t, 0)\right]^{2} h_{-}^{(2)}(0)
\end{aligned}
$$

where we have omitted the boundary terms for the boundary nodes $x=0$ for the first pipe and $x=L_{2}$ for the second pipe since due to our assumptions (5.20), (5.22) on the feedback laws they are negative. So it remains to prove that

$$
B^{(1)}\left[r_{-}^{(1)}\left(t, L_{1}\right)\right]^{2} h_{-}^{(1)}\left(L_{1}\right) \leq A^{(1)}\left[r_{+}^{(1)}\left(t, L_{1}\right)\right]^{2} h_{+}^{(1)}\left(L_{1}\right)
$$


and

$$
A^{(2)}\left[r_{+}^{(2)}(t, 0)\right]^{2} h_{+}^{(2)}(0) \leq B^{(2)}\left[r_{-}^{(2)}(t, 0)\right]^{2} h_{-}^{(2)}(0) .
$$

We start with the functions defined on the first pipe. Since we have

$$
r_{-}^{(1)}\left(t, L_{1}\right)=F_{-}\left(\bar{q}, \bar{R}_{+}^{(1)}\left(L_{1}\right)+r_{+}^{(1)}\left(t, L_{1}\right)\right)-\bar{R}_{-}^{(1)}\left(L_{1}\right)
$$

if $r_{+}^{(1)}\left(t, L_{1}\right)=0$ also $r_{-}^{(1)}\left(t, L_{1}\right)=0$ hence in this case inequality $(5.27)$ holds. If $r_{+}^{(1)}\left(t, L_{1}\right) \neq 0$ inequality $(5.27)$ is equivalent to

$$
\frac{\left[r_{-}^{(1)}\left(t, L_{1}\right)\right]^{2}}{\left[r_{+}^{(1)}\left(t, L_{1}\right)\right]^{2}} \frac{h_{-}^{(1)}\left(L_{1}\right)}{h_{+}^{(1)}\left(L_{1}\right)} \leq \frac{A^{(1)}}{B^{(1)}}
$$

or since $h_{+}^{(1)}\left(L_{1}\right) / h_{-}^{(1)}\left(L_{1}\right)=\exp (-\mu *(1 / \mu))=\exp (-1)$

$$
\frac{\left[r_{-}^{(1)}\left(t, L_{1}\right)\right]^{2}}{\left[r_{+}^{(1)}\left(t, L_{1}\right)\right]^{2}} \leq \exp (-1) \frac{A^{(1)}}{B^{(1)}}
$$

Hence (5.29) is equivalent to

$$
\left[\frac{F_{-}\left(\bar{q}, \bar{R}_{+}^{(1)}\left(L_{1}\right)+r_{+}^{(1)}\left(t, L_{1}\right)\right)-F_{-}\left(\bar{q}, \bar{R}_{+}^{(1)}\left(L_{1}\right)\right)}{r_{+}^{(1)}\left(t, L_{1}\right)}\right]^{2} \leq \exp (-1) \frac{A^{(1)}}{B^{(1)}} .
$$

Due to the mean value theorem we have

$$
\frac{F_{-}\left(\bar{q}, \bar{R}_{+}^{(1)}\left(L_{1}\right)+r_{+}^{(1)}\left(t, L_{1}\right)\right)-F_{-}\left(\bar{q}, \bar{R}_{+}^{(1)}\left(L_{1}\right)\right)}{r_{+}^{(1)}\left(t, L_{1}\right)}=\partial_{R_{+}} F_{-}\left(\bar{q}, \bar{R}_{+}^{(1)}\left(L_{1}\right)+\xi_{+}(t)\right)
$$

for some real number $\xi_{+}(t)$ with $\left|\xi_{+}(t)\right| \leq\left\|r_{+}^{(1)}\left(\cdot, L_{1}\right)\right\|_{C([0, T])}$. Therefore inequality (5.30) holds if $\frac{A^{(1)}}{B^{(1)}}$ is chosen sufficiently large since the function $F_{-}(\bar{q}, \cdot)$ is continuously differentiable and the derivative

$$
\partial_{R_{+}} F_{-}\left(\bar{q}, \bar{R}_{+}^{(1)}\left(L_{1}\right)+\cdot\right)
$$

is uniformly bounded on $[0, T]$ for $r_{+}^{(1)}$ in a sufficiently small $C^{(1)}$ neighbourhood of zero. More precisely, (5.30) holds if $\left\|r_{+}^{(1)}\left(\cdot, L_{1}\right)\right\|_{C([0, T])} \leq \varepsilon$ and

$$
\sup _{\left|\xi_{+}\right| \leq \varepsilon}\left|\partial_{R_{+}} F_{-}\left(\bar{q}, \bar{R}_{+}^{(1)}\left(L_{1}\right)+\xi_{+}\right)\right|^{2} \leq \exp (-1) \frac{A^{(1)}}{B^{(1)}}
$$

Now we come to functions defined on the second pipe. Since we have

$$
r_{+}^{(2)}(t, 0)=F_{+}\left(\bar{q}, \bar{R}_{-}^{(2)}(0)+r_{-}^{(2)}(t, 0)\right)-\bar{R}_{+}^{(2)}(0)
$$

if $r_{-}^{(2)}(t, 0)=0$ also $r_{+}^{(2)}(t, 0)=0$ hence in this case inequality (5.28) holds. If $r_{-}^{(2)}(t, 0) \neq 0$ inequality (5.28) is equivalent to

$$
\frac{\left[r_{+}^{(2)}(t, 0)\right]^{2}}{\left[r_{-}^{(2)}(t, 0)\right]^{2}} \frac{h_{+}^{(2)}(0)}{h_{-}^{(2)}(0)} \leq \frac{B^{(2)}}{A^{(2)}}
$$


or since $h_{+}^{(2)}(0) / h_{-}^{(2)}(0)=1$

$$
\frac{\left[r_{+}^{(2)}(t, 0)\right]^{2}}{\left[r_{-}^{(2)}(t, 0)\right]^{2}} \leq \frac{B^{(2)}}{A^{(2)}}
$$

Hence (5.32) is equivalent to

$$
\left[\frac{F_{+}\left(\bar{q}, \bar{R}_{-}^{(2)}(0)+r_{-}^{(2)}(t, 0)\right)-F_{+}\left(\bar{q}, \bar{R}_{-}^{(2)}(0)\right)}{r_{-}^{(2)}(t, 0)}\right]^{2} \leq \frac{B^{(2)}}{A^{(2)}}
$$

Due to the mean value theorem we have

$$
\frac{F_{+}\left(\bar{q}, \bar{R}_{-}^{(2)}(0)+r_{-}^{(2)}(t, 0)\right)-F_{+}\left(\bar{q}, \bar{R}_{-}^{(2)}(0)\right)}{r_{-}^{(2)}(t, 0)}=\partial_{R_{-}} F_{+}\left(\bar{q}, \bar{R}_{-}^{(2)}(0)+\xi_{-}(t)\right)
$$

for some real number $\xi_{-}(t)$ with $\left|\xi_{-}(t)\right| \leq\left\|r_{-}^{(2)}(\cdot, 0)\right\|_{C([0, T])}$. Therefore equation (5.33) holds if $\frac{B^{(2)}}{A^{(2)}}$ is chosen sufficiently large since the function $F_{+}(\bar{q}, \cdot)$ is continuously differentiable and the derivative $\partial_{R_{-}} F_{+}\left(\bar{q}, \bar{R}_{-}^{(2)}(0)+\cdot\right)$ is uniformly bounded on $[0, T]$ for $r_{-}^{(2)}$ in a sufficiently small $C^{1}$-neighbourhood of zero. More precisely, (5.33) holds if $\left\|r_{-}^{(2)}(\cdot,)\right\|_{C([0, T])} \leq \varepsilon$ and

$$
\sup _{\left|\xi_{-}\right| \leq \varepsilon}\left|\partial_{R_{-}} F_{+}\left(\bar{q}, \bar{R}_{-}^{(2)}(0)+\xi_{-}\right)\right|^{2} \leq \frac{B^{(2)}}{A^{(2)}}
$$

\section{Summary}

We have established an existence result for semi-global classical solutions for a network model of gas dynamics governed by a compressor station. We have given sufficient conditions for the existence of classical stationary states of this system. We have studied the stabilization of the flow of this system by suitable boundary feedback laws and feedback laws at the compressor. In our analysis we have used a Lyapunov function.

The presented results are given for two pipes and a single compressor station. However, the techniques applied can be extended to tree-like networks and more compressor stations. Furthermore, it would be desirable to obtain stabilizability of the system for unbounded time intervals $[0, \infty)$. To this end, the existence result has to be extended to be global in time and different Lyapunov functions have to be studied. This will be dealt with in a forthcoming publication.

Acknowledgements. We thank the DFG SPP 1253, DAAD D/08/11076, HE5386/6-1 and Seed Funds (RWTH Aachen) for financial support. We thank the anonymous referee for helpful comments.

\section{REFERENCES}

[1] M.K. Banda, M. Herty and A. Klar, Coupling conditions for gas networks governed by the isothermal Euler equations. Networks and Heterogenous Media 1 (2006) 295-314.

[2] M.K. Banda, M. Herty and A. Klar, Gas flow in pipeline networks. Networks and Heterogenous Media 1 (2006) 41-56.

[3] C. Bardos, G. Lebeau and J. Rauch, Sharp sufficient conditions for the observation, control, and stabilization of waves from the boundary. SIAM J. Control Optim. 30 (2002) 1024-1065.

[4] G. Bastin, J.-M. Coron and B. d'Andrea-Novel, On Lyapunov stability of linearised Saint-Venant equations for a sloping channel. Networks and Heterogenous Media 4 (2009).

[5] N.H. Chen, An explicit equation for friction factor in pipe. Ind. Eng. Chem. Fund. 18 (1979) 296-297.

[6] R.M. Colombo, G. Guerra, M. Herty and V. Schleper, Optimal control in networks of pipes and canals. SIAM J. Control Optim. 48 (2009) 2032-2050.

[7] J.-M. Coron, Control and Nonlinearity, Mathematical Surveys and Monographs 136. AMS, Providence (2007). 
[8] J.-M. Coron, B. d'Andréa-Novel and G. Bastin, A Lyapunov approach to control irrigation canals modeled by the Saint-Venant equations, in Proc. Eur. Control Conf., Karlsruhe, Germany (1999).

[9] J.-M. Coron, B. d'Andréa-Novel and G. Bastin, On boundary control design for quasi-linear hyperbolic systems with entropies as Lyapunov functions, in Proc. 41st IEEE Conf. Decision Control, Las Vegas, USA (2002).

[10] J.-M. Coron, B. d'Andréa-Novel, G. Bastin and L. Moens, Boundary control for exact cancellation of boundary disturbances in hyperbolic systems of conservation laws, in Proc. 44st IEEE Conf. Decision Control, Seville, Spain (2005).

[11] J.-M. Coron, B. d'Andréa-Novel and G. Bastin, A strict Lyapunov function for boundary control of hyperbolic systems of conservation laws. IEEE Trans. Automat. Contr. 52 (2007) 2-11.

[12] J.-M. Coron, G. Bastin and B. d'Andréa-Novel, Dissipative boundary conditions for one dimensional nonlinear hyperbolic systems. SIAM J. Control Optim. 47 (2008) 1460-1498.

[13] J. de Halleux, C. Prieur, J.-M. Coron, B. d'Andréa-Novel and G. Bastin, Boundary feedback control in networks of open channels. Automatica 39 (2003) 1365-1376.

[14] K. Ehrhardt and M. Steinbach, Nonlinear gas optimization in gas networks, in Modeling, Simulation and Optimization of Complex Processes, H.G. Bock, E. Kostina, H.X. Pu and R. Rannacher Eds., Springer Verlag, Berlin, Germany (2005).

[15] M. Gugat and G. Leugering, Global boundary controllability of the de St. Venant equations between steady states. Ann. Inst. H. Poincaré Anal. Non Linéaire 20 (2003) 1-11.

[16] M. Gugat and G. Leugering, Global boundary controllability of the Saint-Venant system for sloped canals with friction. Ann. Inst. H. Poincaré Anal. Non Linéaire 26 (2009) 257-270.

[17] M. Gugat, G. Leugering and E.J.P.G. Schmidt, Global controllability between steady supercritical flows in channel networks. Math. Meth. Appl. Sci. 27 (2004) 781-802.

[18] M. Herty, Coupling conditions for networked systems of Euler equations. SIAM J. Sci. Comp. 30 (2007) 1596-1612.

[19] M. Herty and V. Sachers, Adjoint calculus for optimization of gas networks. Networks and Heterogeneous Media 2 (2007) $733-750$.

[20] G. Leugering and E.J.P.G. Schmidt, On the modeling and stabilization of flows in networks of open canals. SIAM J. Control Optim. 41 (2002) 164-180.

[21] T.-T. Li, Exact controllability for quasilinear hyperbolic systems and its application to unsteady flows in a network of open canals. Math. Meth. Appl. Sci. 27 (2004) 1089-1114.

[22] T.-T. Li, Exact boundary controllability of unsteady flows in a network of open canals. Math. Nachr. 278 (2005) 310-329.

[23] T.-T. Li and Y. Jin, Semi-global $C^{1}$ solution to the mixed initial-boundary value problem for quasilinear hyperbolic systems. Chin. Ann. Math. B 22 (2001) 325-336.

[24] T.-T. Li and B. Rao, [Exact boundary controllability of unsteady flows in a tree-like network of open canals]. C. R. Acad. Sci. Paris Ser. I 339 (2004) 867-872.

[25] T.-T. Li and Z. Wang, Global exact boundary controllability for first order quasilinear hyperbolic systems of diagonal form. Int. J. Dynamical Systems Differential Equations 1 (2007) 12-19.

[26] T.-T. Li and W.-C. Yu, Boundary value problems for quasilinear hyperbolic systems, Duke University Mathematics Series V. Durham, NC, USA (1985).

[27] A. Martin, M. Möller and S. Moritz, Mixed integer models for the stationary case of gas network optimization. Math. Programming 105 (2006) 563-582.

[28] E. Menon, Gas Pipeline Hydraulics. Taylor and Francis, Boca Raton (2005).

[29] A. Osiadacz, Simulation of transient flow in gas networks. Int. J. Numer. Meth. Fluids 4 (1984) 13-23.

[30] A.J. Osciadacz, Simulation and Analysis of Gas Networks. Gulf Publishing Company, Houston (1987).

[31] A.J. Osciadacz, Different Transient Models - Limitations, advantages and disadvantages, in 28th Annual Meeting of PSIG (Pipeline Simulation Interest Group), San Francisco, California, USA (1996).

[32] Pipeline Simulation Interest Group, www.psig.org.

[33] M. Steinbach, On PDE Solution in Transient Optimization of Gas Networks. Technical Report ZR-04-46, ZIB Berlin, Germany (2004).

[34] Z. Vostrý, Transient Optimization of gas transport and distribution, in Proceedings of the 2nd International Workshop SIMONE on Innovative Approaches to Modelling and Optimal Control of Large Scale Pipelines, Prague, Czech Republic (1993) 53-62.

[35] Z. Wang, Exact controllability for nonautonomous first order quasilinear hyperbolic systems. Chin. Ann. Math. B 27 (2006) $643-656$.

[36] F.M. White, Fluid Mechanics. McGraw-Hill, New York, USA (2002). 